\title{
Exponential integrators for a Markov chain model of the fast sodium channel of cardiomyocytes
}

\author{
Tomáš Starý1 ${ }^{\text {, Vadim N. Biktashev }}{ }^{1}$ \\ ${ }^{1}$ College of Engineering, Mathematics and Physical Sciences, University of Exeter, EX4 4QF, UK
}

\begin{abstract}
The modern Markov chain models of ionic channels in excitable membranes are numerically stiff. The popular numerical methods for these models require very small time steps to ensure stability. Our objective is to formulate and test two methods addressing this issue, so that the timestep can be chosen based on accuracy rather than stability. Both proposed methods extend Rush-Larsen technique, which was originally developed to Hogdkin-Huxley type gate models. One method, "Matrix Rush-Larsen" (MRL) uses a matrix reformulation of the Rush-Larsen scheme, where the matrix exponentials are calculated using precomputed tables of eigenvalues and eigenvectors. The other, "hybrid operator splitting" (HOS) method exploits asymptotic properties of a particular Markov chain model, allowing explicit analytical expressions for the substeps. We test both methods on the Clancy and Rudy (2002) $I_{\mathrm{Na}}$ Markov chain model. With precomputed tables for functions of the transmembrane voltage, both methods are comparable to the forward Euler method in accuracy and computational cost, but allow longer time steps without numerical instability. We conclude that both methods are of practical interest. MRL requires more computations than HOS, but is formulated in general terms which can be readily extended to other Markov Chain channel models, whereas the utility of HOS depends on the asymptotic properties of a particular model. The significance of the methods is that they allow a considerable speed-up of large-scale computations of cardiac excitation models by increasing the time step, while maintaining acceptable accuracy and preserving numerical stability.
\end{abstract}

\section{Index Terms}

Markov chain, ion channel, numerical methods, Rush-Larsen method, exponential time-differentiation, operator splitting

\section{INTRODUCTION}

M ATHEMATICAL models are an essential part of the modern cardiac electrophysiology. They are used for hypothesis testing in research and as a guide for clinical decision. A typical definition of such a model is a high-dimensional (tens of equations) system of ordinary differential equations per excitable unit. Detailed simulations of the heart involve solving such systems for each of millions of cells placed in a mesh representing the cardiac tissue. Such large-scale models can be computationally extremely expensive, hence significant efforts are directed to develop efficient numerical methods for solving such systems.

A typical cardiac excitation model is centered around the Kirchhoff circuit law which gives

$$
I_{\text {stim }}(t)=C \frac{\mathrm{d} V_{m}}{\mathrm{~d} t}+\sum_{\ell} I_{\ell}
$$

where $C$ is the cell membrane's capacitance, $V_{m}=V_{m}(t)$ is the transmembrane potential difference, and $I_{\ell}, \ell=1, \ldots, L$, are currents through ion-specific channels. The currents, in turn, are determined by the Ohm's law,

$$
I_{\ell}=G_{\ell} P_{\ell}(t)\left[V_{m}(t)-E_{\ell}(\vec{X}(t))\right]
$$

where $E_{\ell}(\vec{X})$ is the ion-specific electromotive force, depending on the ionic concentrations $\vec{X}$ via the Nerst equation, $G_{\ell}$ is the total conductance of channels of type $\ell$ when they are all open, and $P_{\ell}$ is the probability of those channels to be open.

The components of the vector $\vec{X}$ are intra- and extra-cellular ionic concentrations, which change in time in the obvious way in accordance with the ionic fluxes and the corresponding volumes; some concentrations in some models are assumed constant. The dynamics of the open probabilities is much more nontrivial, as it reflects the conformation dynamics of the proteins constituting the ion channels .

The classical description of these dynamics, going back to Hodgkin and Huxley (1952) [1], has the form

$$
P_{\ell}(t)=\prod_{i \in \mathcal{I}(\ell)} y_{i}
$$

with a popular, although different from the original Hodgkin and Huxley's, interpretation that the set $\mathcal{I}(\ell)$ corresponds the subunits of the channel, called "gates". These subunits are assumed statistically independent, each of them can be either in 
an "open" or a "closed" state, and the channel is open if and only if each of the subunits is open. Variables $y_{i}$ then are open probabilities of the gates, and their dynamics are described by

$$
\frac{\mathrm{d} y_{i}}{\mathrm{~d} t}=\alpha_{i}\left(V_{m}\right)\left(1-y_{i}\right)-\beta_{i}\left(V_{m}\right) y_{i}
$$

where $\alpha_{i}\left(V_{m}\right)$ are opening rates and $\beta_{i}\left(V_{m}\right)$ are closing rates.

For instance, the original Hodgkin-Huxley description of the fast sodium current $\left(I_{\mathrm{Na}}\right)$ channel uses $\# \mathcal{I}\left(I_{\mathrm{Na}}\right)=4$ gates, three of which, called m-gates, have identical opening $\alpha_{1}\left(V_{m}\right)=\alpha_{2}\left(V_{m}\right)=\alpha_{3}\left(V_{m}\right)=\alpha_{m}\left(V_{m}\right)$ and closing $\beta_{1}\left(V_{m}\right)=$ $\beta_{2}\left(V_{m}\right)=\beta_{3}\left(V_{m}\right)=\beta_{m}\left(V_{m}\right)$ rates, and the fourth, called h-gate, has rates $\alpha_{4}\left(V_{m}\right)=\alpha_{h}\left(V_{m}\right)$ and $\beta_{4}\left(V_{m}\right)=\beta_{h}\left(V_{m}\right)$, hence for this case we have

$$
\begin{aligned}
& P_{I_{\mathrm{Na}}}(t)=m^{3} h, \\
& \frac{\mathrm{d} m}{\mathrm{~d} t}=\alpha_{m}\left(V_{m}\right)(1-m)-\beta_{m}\left(V_{m}\right) m, \\
& \frac{\mathrm{d} h}{\mathrm{~d} t}=\alpha_{h}\left(V_{m}\right)(1-h)-\beta_{h}\left(V_{m}\right) h .
\end{aligned}
$$

A more recent approach is modelling the probabilities of the channel molecules, as a whole, to be in specific conformation states, without the restricting assumptions of statistically independent subunits and only two states for any subunit. This gives generic continuous time Markov chain (MC) models. Let the probability of the $\ell$ 'th channel to be in the $k$ 'th state at time $t$ be $u_{k}(t)$ ("state occupancy"), $k \in \mathcal{K}(\ell), K(\ell)=\# \mathcal{K}(\ell)$, and all such probabilities be considered components of the state (column-) vector $\vec{u}=\left(u_{k}\right)^{\top}=\vec{u}(t)$. Let $g_{k}$ be the relative permeability of the state $k$, then we have

$$
P_{\ell}(t)=\sum_{k=\mathcal{K}(\ell)} g_{k} u_{k}(t)
$$

Typically, $g_{k}=\delta_{k, k^{*}}$ where $k^{*}$ is the "open state". The time evolution of the state vector is described by the system of linear ODEs, known in particular as Kolmogorov (forward) equations, or master equation, of the form

$$
\frac{\mathrm{d} \vec{u}}{\mathrm{~d} t}=\boldsymbol{A}\left(V_{m}\right) \vec{u}
$$

where the non-diagonal components of the matrix $\boldsymbol{A}\left(V_{m}\right)$ are the transition rates (TR) between the states, and the diagonal components are defined by the condition $\sum_{k \in \mathcal{K}} u_{k}=1$ and consequently sum of any column of $\boldsymbol{A}$ should be zero.

The ODE system for cellular membrane can be solved on a computer using standard numerical solvers. A typical solver iteratively computes the states of the system using time-stepping algorithms, that is computing the states at times $t_{n}=t_{0}+n \Delta t$. The size of the time step $\Delta t$ is inversely proportional to the computational cost, measured as CPU time required for the computation. Increasing the time step is a straightforward way of reducing the computational cost.

The maximal acceptable time step is limited by considerations of accuracy and stability (see e.g. [2, Sections 5.10, 5.11]). Whereas the former is "relative" in that it depends on the aims of the research, the latter has a more "absolute" character in that if stability conditions are not satisfied, the solution is unusable for any purpose. Typically, when the time step exceeds the stability limit, the numerical solution is characterized by wild oscillations around the exact solution, and quite often will lead to numerical overflow.

Simple explicit solvers suffer from instabilities the most, and implicit, stable methods, applicable to generic systems of ODEs, are complicated and often costly. The motivation for our research was that taking into account the specific properties of the problem can offer some advantages. Specifically, we have in mind two distinct considerations.

One consideration is that the TRs can range through several orders of magnitude, and some of them can be much faster than other processes described by the excitable cell model. This split of the speeds of the variables suggest a possibility to exploit asymptotic methods.

The other consideration is the linearity of the system (9). Here we are inspired by the example of the exponential integrator algorithm developed by Rush and Larsen in 1978 [3]. It is based on the assumption that the transmembrane voltage, on which the TRs in the gate model (4) depend, changes only slightly during one time step $\Delta t$. So during one time step, the TRs can be approximated by constants, and the equation (4) is then solved analytically. The solution can be conveniently defined in terms of the "steady state" $\bar{y}_{i}=\alpha_{i}\left(V_{m}\right) /\left[\alpha_{i}\left(V_{m}\right)+\beta_{i}\left(V_{m}\right)\right]$ and the "time constant" $\tau_{i}=1 /\left[\alpha_{i}\left(V_{m}\right)+\beta_{i}\left(V_{m}\right)\right]$ at a given potential $V_{m}$ presumed constant:

$$
y_{i}^{n+1}=\bar{y}_{i}\left(V_{m}\right)-\left[\bar{y}_{i}\left(V_{m}\right)-y_{i}^{n}\right] \exp \left(-\frac{\Delta t}{\tau_{i}\left(V_{m}\right)}\right) .
$$

The Rush-Larsen (RL) scheme is easy to implement, gives good results and is very popular in computational cardiac electrophysiology. Its stability and approximation properties have been extensively discussed in literature, including its relation to general exponential integrators family, its extension beyond gating variables by linearization, and improving its approximation properties, see e.g. [4], [5], [6]. However it is designed for a single ODE and is not immediately applicable for MC models 
TABLE I

CHANGE OF STATES VARIABLES TERMINOLOGY.

\begin{tabular}{lll}
\hline Standard & Our & initial value \\
\hline$O$ & $O$ & $4.386 \times 10^{-8}$ \\
$\mathrm{C}_{1}$ & $P$ & $5.329 \times 10^{-5}$ \\
$\mathrm{C}_{2}$ & $Q$ & $1.064 \times 10^{-2}$ \\
$\mathrm{C}_{3}$ & $R$ & $8.018 \times 10^{-1}$ \\
$\mathrm{IC}_{3}$ & $S$ & $1.436 \times 10^{-1}$ \\
$\mathrm{IC}_{2}$ & $T$ & $1.907 \times 10^{-3}$ \\
$\mathrm{IF}$ & $U$ & $1.111 \times 10^{-5}$ \\
$\mathrm{IM}_{1}$ & $V$ & $8.417 \times 10^{-4}$ \\
$\mathrm{IM}_{2}$ & $W$ & $4.118 \times 10^{-2}$ \\
\hline
\end{tabular}

which are systems of coupled ODEs. And yet MC models are known to suffer from severe numerical instability issues, just as, or even more than, the gate models (Fig. 1). The classical techniques for numerical solution of continuous-time MC models involve finding the eigenvalues and associated eigenvectors of the transition matrix. Direct implementation of this approach to very large MCs is problematic, see e.g. [7]. However the MCs describing ionic channels are relatively small so the direct approach is feasible.

In this paper, we discuss two methods for numerical solution of MC models based on these two considerations.

\section{MethodS}

\section{A. Models}

To test the suggested numerical methods we have chosen the MC model of the $I_{\mathrm{Na}}$ channel by Clancy and Rudy [8] (Fig. 2), which is one of the most popular MC models. We used the formulation of the MC model and the whole cell model into which it was incorporated, as implemented in the authors' code kindly provided by C.E. Clancy. It most closely corresponds to the LuoRudy model [9] with modifications described in [10], [11], and some further minor differences. For the sake of reproducibility of our results, we describe the whole model in the supplementary material, highlighting all the differences from the published models that we have detected. For the same purpose, we put a simplified version of the $\mathrm{C}$ code we used in the simulations described below in the supplementary materials.

For convenience, we changed the notation for the MC states and TRs. The states were named in alphabetical order, starting with $O$ for the open state, in a clockwise direction as in Fig. 2. See Tab. I and Tab. II for the correspondence with the original notation. The model contains 9 interconnected states. The state $O$ represents the conformation of the ion channel that allows the flow of ions between the intracellular and extracellular environment. The remaining states $(P, Q, R, S, T, U, V$ and $W)$ represent non-conductive conformations of the channel, so we can say that for this model $g_{k}=\delta_{k, 1}$, where $u_{1}=O$. There are 11 possible bidirectional transitions between states, but some of the corresponding 22 TRs are described by identical functions,
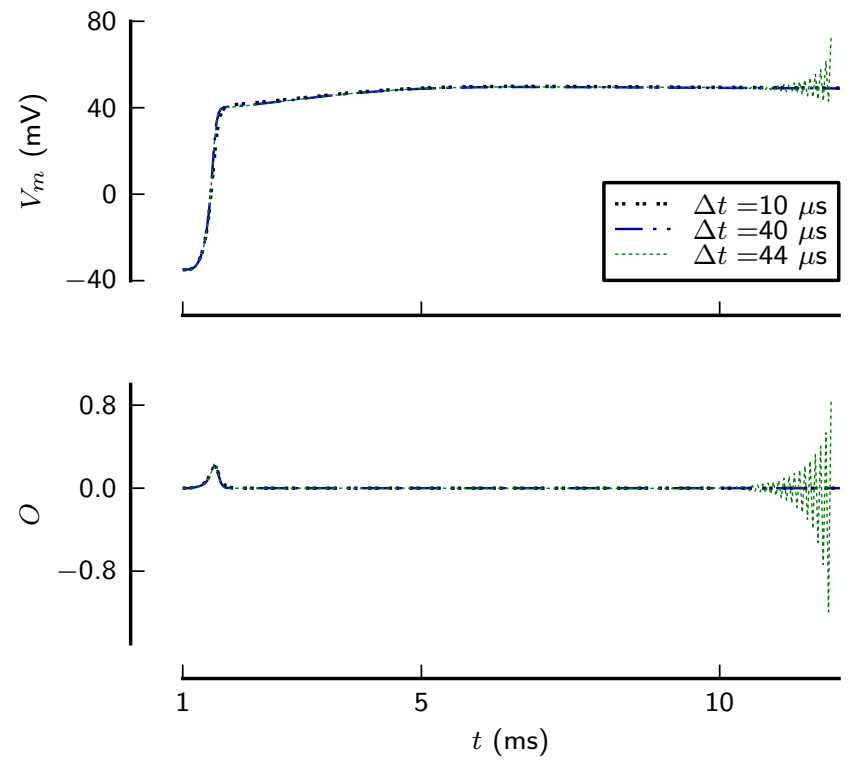

Fig. 1. Instability of $I_{\mathrm{Na}}$ Markov chain model at longer time steps. The model was solved using forward Euler method using three different step sizes: $\Delta t=10 \mu \mathrm{s}, \Delta t=40 \mu \mathrm{s}$, and $\Delta t=44 \mu \mathrm{s}$. The top panel shows the membrane potential $\left(V_{m}\right)$, the bottom panel shows state occupancy of the open state $O$. 


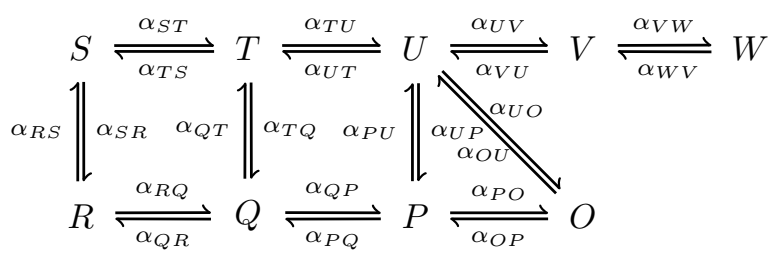

Fig. 2. Markov chain model of $I_{\mathrm{Na}}$ channel.

TABLE II

CHANGE OF TRANSITION RATES (TR) TERMINOLOGY.

\begin{tabular}{ll}
\hline Standard & Our \\
\hline$\alpha_{11}$ & $\alpha_{R Q}, \alpha_{S T}$ \\
$\alpha_{12}$ & $\alpha_{Q P}, \alpha_{T U}$ \\
$\alpha_{13}$ & $\alpha_{P O}$ \\
$\beta_{11}$ & $\alpha_{Q R}, \alpha_{T S}$ \\
$\beta_{12}$ & $\alpha_{P Q}, \alpha_{U T}$ \\
$\beta_{13}$ & $\alpha_{O P}$ \\
$\alpha_{2}$ & $\alpha_{O U}$ \\
$\beta_{2}$ & $\alpha_{U O}$ \\
$\alpha_{3}$ & $\alpha_{U P}, \alpha_{T Q}, \alpha_{S R}$ \\
$\beta_{3}$ & $\alpha_{P U}, \alpha_{Q T}, \alpha_{R S}$ \\
$\alpha_{4}$ & $\alpha_{U V}$ \\
$\beta_{4}$ & $\alpha_{V U}$ \\
$\alpha_{5}$ & $\alpha_{V W}$ \\
$\beta_{5}$ & $\alpha_{W V}$ \\
\hline
\end{tabular}

so there are only 14 distinct TR definitions. We denote the TRs by $\alpha$ with a subscript showing the direction of the transition, e.g. $\alpha_{P O}$ is the transition rate from state $P$ into state $O$. See Tab. II for the link with the original notations.

The TRs are shown on Fig. 3 as functions of the transmembrane potential $V_{m}$ in a physiologically relevant range. The values of TRs vary across several orders of magnitude, from $10^{-11} \mathrm{~ms}^{-1}$ to $10^{2} \mathrm{~ms}^{-1}$. Some of the TRs are high at the lower potentials, some are fast at higher potentials, and some are uniformly low.

The conductive (open) state $O$ is the only state that has immediate effect on the $I_{\mathrm{Na}}$ current. The remaining 8 states of the model can affect the current only indirectly by transitions to the open state $O$. The time evolution of a generic state occupancy state $u_{k}$ is described by a differential equation of the form

$$
\frac{\mathrm{d} u_{k}}{\mathrm{~d} t}=\sum_{k^{\prime} \in \mathcal{K}^{\prime}(k)}\left(\alpha_{k^{\prime}, k} u_{k^{\prime}}-\alpha_{k, k^{\prime}} u_{k}\right),
$$

where $\mathcal{K}^{\prime}(k)$ is the set of all the states interconnected with state $k$, which can be readily found from the diagram. For example, the occupancy of the open state, $O$, is described by the following equation:

$$
\frac{\mathrm{d} O}{\mathrm{~d} t}=\alpha_{P O} P+\alpha_{U O} U-\left(\alpha_{O P}+\alpha_{O U}\right) O .
$$

By taking the sum of the equations (11) for all $k$, one can see that the sum of the right-hand sides equals to zero, and therefore the system observes a states conservation law, which is consistent with the definition of $u_{k}$ as probabilities, implying
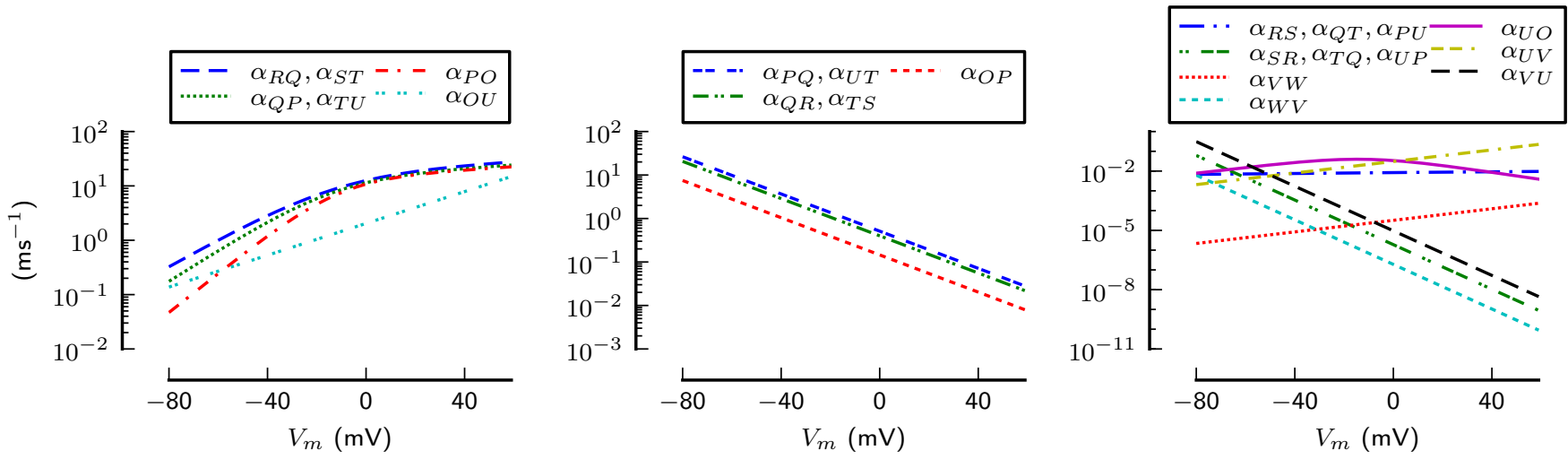

Fig. 3. Transition rates (TR) of $I_{\mathrm{Na}}$ Markov chain model. The left panel shows fast TR at high potentials: subsystem $\boldsymbol{A}_{0}$; the middle panel shows fast TR at low potentials: subsystem $\boldsymbol{A}_{1}$; and the right panel shows slow TR in the entire range of potentials: subsystem $\boldsymbol{A}_{2}$. 
$\sum_{k \in \mathcal{K}} u_{k}=1$. This is of course a generic property of a continuous Markov chain. So the differential equations in the model are not independent, which creates a possibility of reducing the number of equations from 9 to 8 , by computing one of the occupancies through the conservation law rather than from its differential equation. However the computational gain from this is insignificant, and instead we used any deviations from the conservation law as an indicator of the accuracy of the computations.

\section{B. Numerical Methods}

\section{1) Forward Euler}

The standard forward Euler (FE) method is the simplest timestepper for differential equations. It defines the solution at the next time step, $\vec{u}_{n+1}=\vec{u}\left(t_{n+1}\right)$, in terms of the same at the previous time step, $\vec{u}_{n}=\vec{u}\left(t_{n}\right)$, using one-step forward-time finite different approximation of the time derivative, which for the system (9) gives

$$
\vec{u}_{n+1}=\vec{u}_{n}+\Delta t \boldsymbol{A}\left(V_{m}\left(t_{n}\right)\right) \vec{u}_{n} .
$$

The time discretization step $\Delta t=t_{n+1}-t_{n}$ is presumed here the same for all steps of a simulation.

\section{2) Matrix Rush-Larsen}

The proposed Matrix Rush-Larsen method (MRL) assumes that the matrix $\boldsymbol{A}\left(V_{m}\right)$ changes only slightly during one time step and therefore can be approximated by a constant. The solution of (9) can then be written in terms of the matrix exponential,

$$
\vec{u}_{n+1}=\exp \left[\boldsymbol{A}\left(V_{m}\left(t_{n}\right)\right) \Delta t\right] \vec{u}_{n} .
$$

We assume that the matrix $\boldsymbol{A}\left(V_{m}\right)$ is diagonalizable, i.e. can be represented in the form $\boldsymbol{A}\left(V_{m}\right)=\boldsymbol{S}\left(V_{m}\right) \boldsymbol{\Lambda}\left(V_{m}\right) \boldsymbol{S}\left(V_{m}\right)^{-1}$, where matrix $\boldsymbol{S}\left(V_{m}\right)$ is composed of the eigenvectors concatenated as column vectors, and matrix $\boldsymbol{\Lambda}\left(V_{m}\right)$ contains eigenvalues placed on the corresponding places on the diagonal. A sufficient condition of diagonalizability of a matrix is that all its eigenvalues are distinct, and this is the generic situation; but we of course check that it actually takes place in every case. Then the matrix exponential is calculated as

$$
\vec{u}_{n+1}=\boldsymbol{S}\left(V_{m}\right) \exp \left(\boldsymbol{\Lambda}\left(V_{m}\right) \Delta t\right) \boldsymbol{S}\left(V_{m}\right)^{-1} \vec{u}_{n},
$$

where the exponential $\exp \left[\boldsymbol{\Lambda}\left(V_{m}\right) \Delta t\right]$ of the diagonal matrix $\boldsymbol{\Lambda}\left(V_{m}\right) \Delta t$ is obtained by exponentiation of its diagonal elements.

As the numerical solution of the eigenvalue problem is computationally expensive, we precompute the matrices $\boldsymbol{S}\left(\tilde{V}_{j}\right)$, $\boldsymbol{S}\left(\tilde{V}_{j}\right)^{-1}$ and $\boldsymbol{\Lambda}\left(\tilde{V}_{j}\right)$ for a fine grid of physiological potentials, $\tilde{V}_{j}=V_{\min }+j \Delta V, j \in \mathcal{J}=\left\{0,1, \ldots j_{\max }\right\}, \tilde{V}_{j} \leq V_{\max }$, $V_{\min }=-100, V_{\max }=70, \Delta V=0.01$ (all in $\mathrm{mV}$ ) before compile time and save them in a file.

At start time, the eigenvalue and eigenvector matrices are loaded from the file and we precompute, for $\Delta t$ used in the particular simulation, the transition matrices

$$
\boldsymbol{T}_{j}=\boldsymbol{T}\left(\tilde{V}_{j}\right)=\boldsymbol{S}\left(\tilde{V}_{j}\right) \exp \left[\boldsymbol{\Lambda}\left(\tilde{V}_{j}\right) \Delta t\right] \boldsymbol{S}\left(\tilde{V}_{j}\right)^{-1}
$$

for all $j \in \mathcal{J}$. At the run time, the solver simply refers to the tabulated transition matrix $\boldsymbol{T}_{j}$,

$$
\vec{u}_{n+1}=\boldsymbol{T}_{j(n)} \vec{u}_{n}
$$

where $\tilde{V}_{j(n)}$ is the tabulated transmembrane potential that is the nearest to $V_{m}\left(t_{n}\right)$.

Along with the code, we provide precomputed files for a voltage step size of $\Delta V=0.1$ (size of $4.85 \mathrm{MB}$ ), that are sufficient to obtain accurate results. The tables with $\Delta V=0.01 \mathrm{mV}$ of $48.5 \mathrm{MB}$ size, used for the simulations presented, are available from the authors upon request.

The method of tabulation (tab.) can be applied to all the presented numerical methods. However, its benefit is most essential in the MRL method, because matrix exponentiation is computationally expensive. The accuracy of the tabulation is dependent on the voltage step (here $0.01 \mathrm{mV}$ ) which is a matter of choice depending on memory availability and allowable pre-compile and start-time computation time.

\section{3) Hybrid Operator Splitting}

The MRL achieves the purpose in principle but is relatively costly as multiplication by a dense $K \times K$ matrix $\boldsymbol{T}_{j(n)}$ is required at each time step. On the other hand, it did not at all exploit the specific structure of the TR, illustrated by Fig. 3, that is, that the matrix $\boldsymbol{A}\left(V_{m}\right)$ is sparse and some TRs are much faster than others for some voltage ranges. Hence we propose a hybrid operator splitting method (HOS), which combines FE and MRL, and exploits the asymptotic structure of the TRs. In this method, we set

$$
\boldsymbol{A}\left(V_{m}\right)=\boldsymbol{A}_{0}\left(V_{m}\right)+\boldsymbol{A}_{1}\left(V_{m}\right)+\boldsymbol{A}_{2}\left(V_{m}\right)
$$

as described in Fig. 3: $\boldsymbol{A}_{0}$ contains only TRs that are fast at high values of $V_{m}\left(\alpha_{R Q}, \alpha_{S T}, \alpha_{Q P}, \alpha_{T U}, \alpha_{P O}\right.$ and $\left.\alpha_{O U}\right) ; \boldsymbol{A}_{1}$ contains only TRs that are fast at low values of $V_{m}\left(\alpha_{P Q}, \alpha_{U T}, \alpha_{Q R}, \alpha_{T S}\right.$ and $\left.\alpha_{O P}\right)$; and $\boldsymbol{A}_{2}$ contains only uniformly slow 
TRs $\left(\alpha_{R S}, \alpha_{Q T}, \alpha_{P U}, \alpha_{S R}, \alpha_{T Q}, \alpha_{U P}, \alpha_{V W}, \alpha_{W V}, \alpha_{U O}, \alpha_{U V}\right.$ and $\left.\alpha_{V U}\right)$. Explicit expressions for $\boldsymbol{A}_{j}$ are given in the Supplement.

Every timestep is then done in three substeps,

$$
\begin{aligned}
\vec{u}_{n+1 / 3} & =\exp \left(\Delta t \boldsymbol{A}_{0}\left(V_{m}\left(t_{n}\right)\right)\right) \vec{u}_{n}, \\
\vec{u}_{n+2 / 3} & =\exp \left(\Delta t \boldsymbol{A}_{1}\left(V_{m}\left(t_{n}\right)\right)\right) \vec{u}_{n+1 / 3}, \\
\vec{u}_{n+1} & =\vec{u}_{n+2 / 3}+\Delta t \boldsymbol{A}_{2}\left(V_{m}\left(t_{n}\right)\right) \vec{u}_{n+2 / 3} .
\end{aligned}
$$

In our case the matrix exponentials in the two fast subsystems (17) and (18) are found analytically, through solving the corresponding ODE systems. This is possible because some of the equations corresponding to the matrices $\boldsymbol{A}_{0}\left(V_{m}\right)$ and $\boldsymbol{A}_{1}\left(V_{m}\right)$ are coupled in a specific manner and can be solved one by one where solution of one equation is substituted in the next etc. The full expressions and the method of derivation are given in the Supplement; here we present the solution for state $O$ in the equation (17) as an example:

$$
O_{n+1 / 3}=\mu_{O U} O_{n}+K_{P O} P_{n}+K_{Q O} Q_{n}+K_{R O} R_{n}
$$

where

$$
\begin{aligned}
K_{P O}= & \frac{\alpha_{P O}\left(\mu_{P O}-\mu_{O U}\right)}{\alpha_{O U}-\alpha_{P O}}, \\
K_{Q O}= & \frac{\alpha_{P O} \alpha_{Q P}\left(\mu_{Q P}-\mu_{O U}\right)}{\left(\alpha_{P O}-\alpha_{Q P}\right)\left(\alpha_{O U}-\alpha_{Q P}\right)} \\
& -\frac{\alpha_{P O} \alpha_{Q P}\left(\mu_{P O}-\mu_{O U}\right)}{\left(\alpha_{P O}-\alpha_{Q P}\right)\left(\alpha_{O U}-\alpha_{P O}\right)}, \\
K_{R O}= & -\frac{\alpha_{P O} \alpha_{Q P} \alpha_{R Q}\left(\mu_{Q P}-\mu_{O U}\right)}{\left(\alpha_{Q P}-\alpha_{R Q}\right)\left(\alpha_{P O}-\alpha_{Q P}\right)\left(\alpha_{O U}-\alpha_{Q P}\right)} \\
& +\frac{\alpha_{P O} \alpha_{Q P} \alpha_{R Q}\left(\mu_{P O}-\mu_{O U}\right)}{\left(\alpha_{Q P}-\alpha_{R Q}\right)\left(\alpha_{P O}-\alpha_{Q P}\right)\left(\alpha_{O U}-\alpha_{P O}\right)} \\
& +\frac{\alpha_{P O} \alpha_{Q P} \alpha_{R Q}\left(\mu_{R Q}-\mu_{O U}\right)}{\left(\alpha_{Q P}-\alpha_{R Q}\right)\left(\alpha_{P O}-\alpha_{R Q}\right)\left(\alpha_{O U}-\alpha_{R Q}\right)} \\
& -\frac{\alpha_{P O} \alpha_{Q P} \alpha_{R Q}\left(\mu_{P O}-\mu_{O U}\right)}{\left(\alpha_{Q P}-\alpha_{R Q}\right)\left(\alpha_{P O}-\alpha_{R Q}\right)\left(\alpha_{O U}-\alpha_{P O}\right)}, \\
\mu_{j k}= & \mathrm{e}^{-\alpha_{j k} \Delta t} .
\end{aligned}
$$

The slow subsystem (19) uses FE, and since it contains only uniformly slow TRs, it can tolerate large time-steps, allowed by other components of the cell model, without loss of stability.

\section{A priori error estimates}

Estimates by standard methods (e.g. [2, Chapter 5], see details in the Supplement), show that all three numerical schemes have local truncation errors of the second order, i.e. $\mathcal{E} \Delta t^{2}+\mathcal{O}\left(\Delta t^{3}\right)$, although the coefficients $\mathcal{E}$ vary: for FE we have $\mathcal{E}_{\mathrm{FE}} \leq$ $\frac{1}{2}\left(\|\boldsymbol{A}\|^{2}+\left\|\mathrm{d} \boldsymbol{A} / \mathrm{d} V_{m}\right\|\left|\mathrm{d} V_{m} / \mathrm{d} t\right|\right)$, for MRL we have $\mathcal{E}_{\mathrm{MRL}}=\frac{1}{2}\left\|\mathrm{~d} \boldsymbol{A} / \mathrm{d} V_{m}\right\|\left|\mathrm{d} V_{m} / \mathrm{d} t\right|$, and for HOS it is composed of contributions of the three substeps plus the error due to operator splitting, $\mathcal{E}_{\mathrm{HOS}} \leq \frac{1}{2}\left|\mathrm{~d} V_{m} / \mathrm{d} t\right|\left(\left\|\mathrm{d} \boldsymbol{A}_{0} / \mathrm{d} V_{m}\right\|+\left\|\mathrm{d} \boldsymbol{A}_{1} / \mathrm{d} V_{m}\right\|+\left\|\mathrm{d} \boldsymbol{A}_{2} / \mathrm{d} V_{m}\right\|\right)+$ $\frac{1}{2}\left\|\boldsymbol{A}_{2}\right\|^{2}+\mathcal{E}_{\mathrm{OS}}$, where $\mathcal{E}_{\mathrm{OS}}=\frac{1}{2}\left\|\left[\boldsymbol{A}_{1}, \boldsymbol{A}_{0}\right]+\left[\boldsymbol{A}_{2}, \boldsymbol{A}_{0}\right]+\left[\boldsymbol{A}_{2}, \boldsymbol{A}_{1}\right]\right\|$, and $[\boldsymbol{X}, \boldsymbol{Y}] \equiv \boldsymbol{X} \boldsymbol{Y}-\boldsymbol{Y} \boldsymbol{X}$. So comparison of MRL and HOS with FE depends on the solution, but in any case accuracy of HOS it contingent on $\boldsymbol{A}_{0}, \boldsymbol{A}_{1}$ and $\boldsymbol{A}_{2}$ not being large at the same time, to ensure relative smallness of $\mathcal{E}_{\mathrm{OS}}$.

\section{Implementation}

Most of the algorithms described here were implemented in $\mathrm{C}$ language in double precision floating point arithmetics and compiled using GNU Compiler Collection (GCC) (version 4.7.2). The exception is computation of eigenvalues and eigenvector tables, which was done using mathematical software Sage [12] (version 5.9). Simulation were performed on Intel Core i5-3470 CPU with the clock frequency 3.20GHz under GNU/Linux operating system (distribution Fedora 18).

\section{RESULTS}

Figure 4 shows the detail of the first millisecond of simulated cardiac excitation, the onset of an action potential (AP). The $I_{\mathrm{Na}}$ Markov chain model was solved using the three suggested integration methods: forward Euler (FE), matrix RushLarsen (MRL), and hybrid operator splitting (HOS), as described in the methods section. The model was solved with time step 

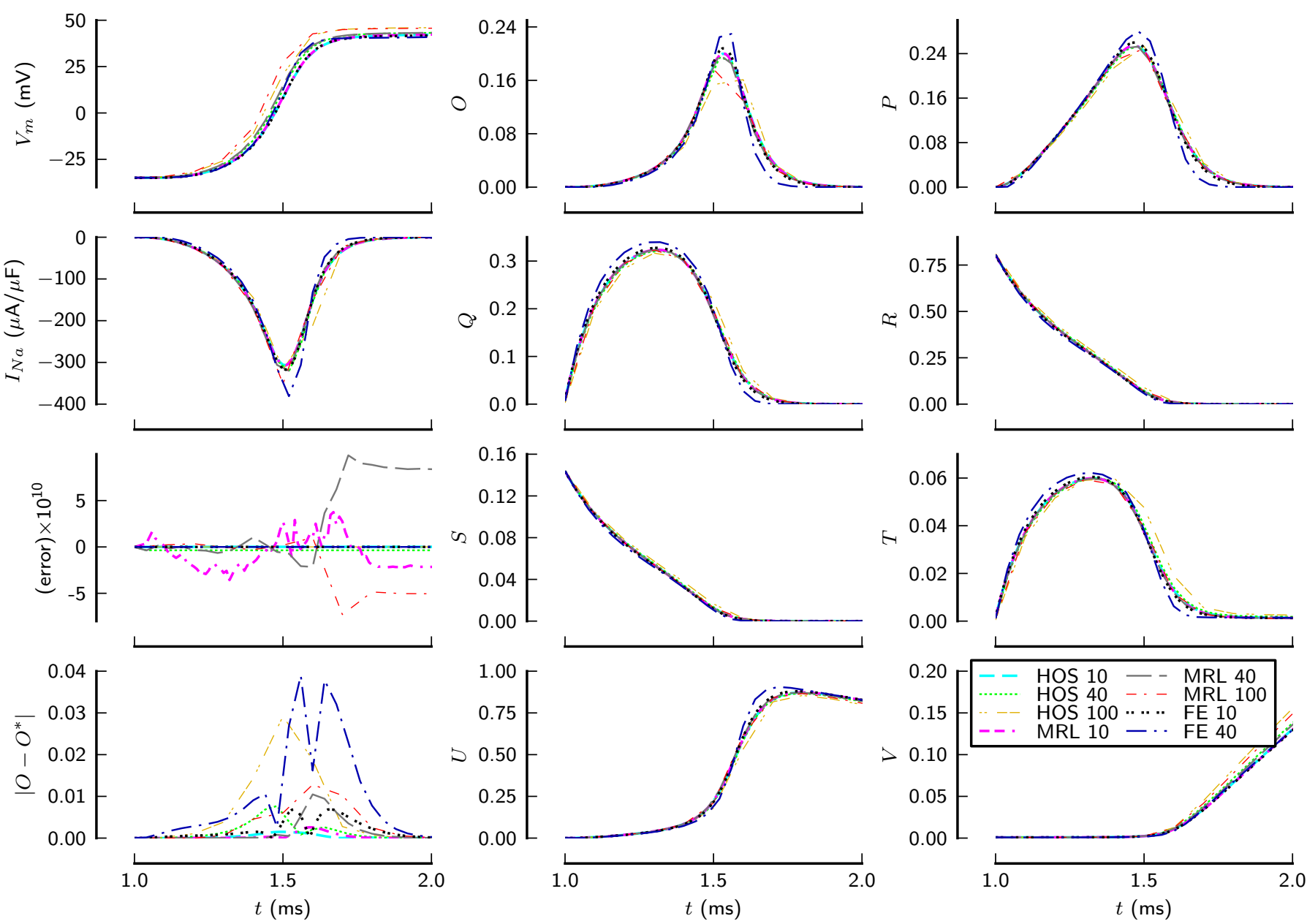

Fig. 4. Cardiac cell action potential (AP) simulations (detail of the first second) with $I_{\mathrm{Na}}$ Markov chain model solved using: hybrid operator splitting method (HOS) - light colours; matrix Rush-Larsen (MRL) - semi-dark colours; and forward Euler (FE) - dark colours; The left column shows: membrane potential $\left(V_{m}\right)$, sodium current $\left(I_{\mathrm{Na}}\right)$, error calculated as deviation from the states conservation law, and deviation of the state occupancy $O$ from the solution using FE with $\Delta t=1 \mu \mathrm{s}\left(O^{*}\right)$; the other two columns show the state occupancies. The model was solved with time step $\Delta t=10 \mu \mathrm{s}, \Delta t=40 \mu \mathrm{s}$, and $\Delta t=100 \mu \mathrm{s}$ represented by thick, middle thick, thin lines respectively.

$\Delta t=10 \mu \mathrm{s}, \Delta t=40 \mu \mathrm{s}$, and $\Delta t=100 \mu \mathrm{s}$, except for FE, which was also solved for $\Delta t=1 \mu \mathrm{s}$, to be used as a reference, but not for $\Delta t=100 \mu \mathrm{s}$, due to instability.

The model excitable cell was initially at the resting state, and at the time $t=1 \mathrm{~ms}$, an AP was initialized by instantaneous injection of potassium ions, raising the membrane potential to $V_{m}=-35 \mathrm{mV}$. The initial conditions of the states of the $I_{\mathrm{Na}} \mathrm{MC}$ model are specified in Tab. I and the initial states of the remaining variables of the model can be found in the supplementary material. Before the initiation, more than $90 \%$ of the channels reside in the states $R$ and $S$, which require at least three transitions to get to the open state. After the initiation, the channels start to transit rapidly towards the open state $O$ and then to the state $U$. Within about $0.7 \mathrm{~ms}$ almost all channels reside in the state $U$. Then, the channels slowly transit to the state $V$, where they stay until the resting potential $V_{m}$ is recovered. The states $U$ and $V$ are similar to the situation when the inactivation gate $h$ is closed in the gate model. The states $S$ and $T$ have less than $10 \%$ occupancy during all the stages of the action potential.The plot of $W$ is omitted, as this variable changes very little during the time interval shown.

The results for the time step $\Delta t=10 \mu \mathrm{s}$ are consistent in all panels. The FE is still stable at time step $\Delta t=40 \mu \mathrm{s}$, however, compared to MRL and HOS, the FE solution is less accurate, resulting in a higher peak and faster decay of both the open state $O$ occupancy and the resulting $I_{\mathrm{Na}}$ current.

Comparison of the solutions for $O(t)$ with the reference $O^{*}(t)$, obtained by FE with $\Delta t=1 \mu \mathrm{s}$, is shown on Fig. 4 (first column, fourth row). We see that MRL and HOS approximate $O^{*}$ better than FE at the same time steps. This is consistent with results of evaluation of the error estimates over the AP solution: we have $\max \left(\mathcal{E}_{\mathrm{FE}}\right) \approx 2700, \max \left(\mathcal{E}_{\mathrm{MRL}}\right) \approx$ $118, \max \left(\mathcal{E}_{\mathrm{HOS}}\right) \approx 125$, with $\max \left(\mathcal{E}_{\mathrm{OS}}\right) \approx 19$, all in $\mathrm{ms}^{-2}$, and $\min \left(\mathcal{E}_{\mathrm{FE}} / \mathcal{E}_{\mathrm{HOS}}\right) \approx 2.3, \min \left(\mathcal{E}_{\mathrm{FE}} / \mathcal{E}_{\mathrm{MRL}}\right) \approx 3.2$ (see the Supplement). This suggests that exponential integrators can be useful, for their accuracy, even when instability is not a concern, say in systems with slower dynamics, such as $I_{\mathrm{Ks}}$.

At longer time steps, FE is unstable (Fig. 1 illustrates a mild case of the instability), while MRL and HOS continue to 
TABLE III

Elapsed Simulation time [S]. Cell model [8] 100 PUlSes With CL=1000 MS.

\begin{tabular}{l||cc|cc|cc}
\hline \multirow{2}{*}{$I_{\text {Na Model }}$} & \multicolumn{2}{c|}{$\Delta t=10 \mu \mathrm{s}$} & \multicolumn{2}{c|}{$\Delta t=40 \mu \mathrm{s}$} & \multicolumn{2}{c}{$\Delta t=100 \mu \mathrm{s}$} \\
\cline { 2 - 7 } & $I_{\mathrm{Na}}$ & Total & $I_{\mathrm{Na}}$ & Total & $I_{\mathrm{Na}}$ & Total \\
\hline FE & 4.88 & 22.34 & 1.24 & 5.59 & & \\
FE (tab.) & 2.48 & 19.98 & 0.60 & 5.01 & & \\
MRL (tab.) & 2.96 & 20.45 & 0.74 & 5.16 & 0.28 & 2.06 \\
HOS & 8.11 & 25.71 & 2.01 & 6.43 & 0.81 & 2.58 \\
HOS (tab.) & 2.81 & 20.31 & 0.71 & 5.11 & 0.29 & 2.05 \\
\hline
\end{tabular}

provide stable solutions. At $\Delta t=100 \mu \mathrm{s}$, the peak of the most important component of $\vec{u}$, the occupancy of the open state $O$, is slightly lower than at shorter timesteps. On the other hand, the decrease of the peak of the total $I_{\mathrm{Na}}$ current in these two methods (HOS, MRL at $\Delta t=100 \mu \mathrm{s}$ ) is relatively small compared to the decrease of the open state occupancy. Also, the decay of the $I_{\mathrm{Na}}$ current in the MRL $\Delta t=100 \mu \mathrm{s}$ is slower than in the other cases. Note that the lead of the APs onsets at $\Delta t=100 \mu \mathrm{s}$ against smaller time steps is comparable to the value of $\Delta t$.

Approximation of the whole APs rather than just their onsets is illustrated in Fig. 5.

Further increase of the time steps (not shown) in MRL and HOS gives significant errors in the AP, e.g. at $\Delta t=200 \mu$ s there is a $30 \mathrm{mV}$ overshoot. Stability persists for much longer: for HOS the solution becomes unphysical (a negative concentration) at about $\Delta t=2 \mathrm{~ms}$ without loss of stability, and for MRL an instability occurs at about $7.5 \mathrm{~ms}$, although the solution is then also very different from the true AP.

Table III illustrates the efficiency of the three methods at three different time steps $\Delta t$. This was done by measuring time taken by simulations consisting of 100 pulses with a cycle length (CL) of $1000 \mathrm{~ms}$ without any output. The pulses were initialized by an instantaneous injection of potassium ions of a sufficient amount to set the membrane potential to $V_{m}=-35 \mathrm{mV}$. The table shows times taken by the whole cell model ("Total") and by the Markov Chain model computations (" $I_{\mathrm{Na}}$ "). The times shown are median values from six separate simulations in each case to minimize the effect of other processes running on the computer.

At $\Delta t=10 \mu \mathrm{s}, \mathrm{FE}$ is the most efficient method. Computation of $I_{\mathrm{Na}}$ accounts for $21.8 \%$ and $31.5 \%$ of the overall computation cost in FE and HOS respectively. Tabulation allows reduction of the computation cost of $I_{\mathrm{Na}}$ by $49.1 \%$ and $65.3 \%$ in FE and HOS. MRL was used only with tabulation using the precomputed eigenvalues and eigenvectors matrices and the computational cost at the $\Delta t=10 \mu \mathrm{s}$ is comparable with FE. These proportions are consistent with the results at time step $\Delta t=40 \mu \mathrm{s}$ and $\Delta t=100 \mu \mathrm{s}$ for MRL and HOS. So, at the same time step, the computational costs of the proposed methods are slightly higher, but the accuracies are somewhat better, compared to FE. The most important benefit of HOS and MRL is, however, the possibility of using larger time steps.

\section{CONCLUSION}

Both proposed methods maintain stability at larger time steps, and improve the accuracy of the solution at the same time step, compared to the explicit ODE solver (FE). When tabulated, those methods are comparable to FE in computational cost. As expected, using larger time steps results in reduction of computational cost.

MRL method extends the popular RL method, developed for gate models, to Markov chain models. MRL is more universal than HOS, and may be made "automatic". The only restriction of our implementation is the assumption of diagonalizability of matrix $\boldsymbol{A}\left(V_{m}\right)$ for all voltages. If in another model this happens not to be the case, then some more sophisticated approach would be needed. If non-diagonalizability is a regular feature, say due to identical definitions of some of the TRs, then a Jordan form can be used instead; if it only happens at selected voltages, then interpolation of matrices $\boldsymbol{T}\left(V_{m}\right)$ may be sufficient.

HOS method depends on the possibility to split the transition rates to multiple (three in our case) sub-systems according to their speeds, and solve each of the subsystems on its own. Our solution benefits from the possibility of solving the fast subsystems analytically. Implementing the analytical solution results in even better speed-up as the resulting timestepping matrices are sparse. However, the possibility of a suitable analytical solution is not guaranteed for a general MC model. In this case, the fast time subsystems can be solved using diagonalization like in the MRL method, which might require additional computational time.

Finally we comment on the order of approximation. In this paper we considered first order schemes, and they are most popular in practice. However, the approximation order can be improved by using more sophisticated methods, both for the whole cell model (say using Runge-Kutta approach) and for the exponential solvers. For the original Rush-Larsen scheme, higher-order variants have been proposed and tested [4], [5], and the same ideas can be extended to the matrix case as well. Naturally, HOS method may then need to involve a more sophisticated operator splitting method to correspond.

Another appealing direction for further research is application of the proposed methods to other important MC models. MRL is straightforward for any MC where TRs depend only on one variable, otherwise tabulation will be a bit more problematic. The success of the HOS approach will depend on the asymptotic properties of the TRs. 

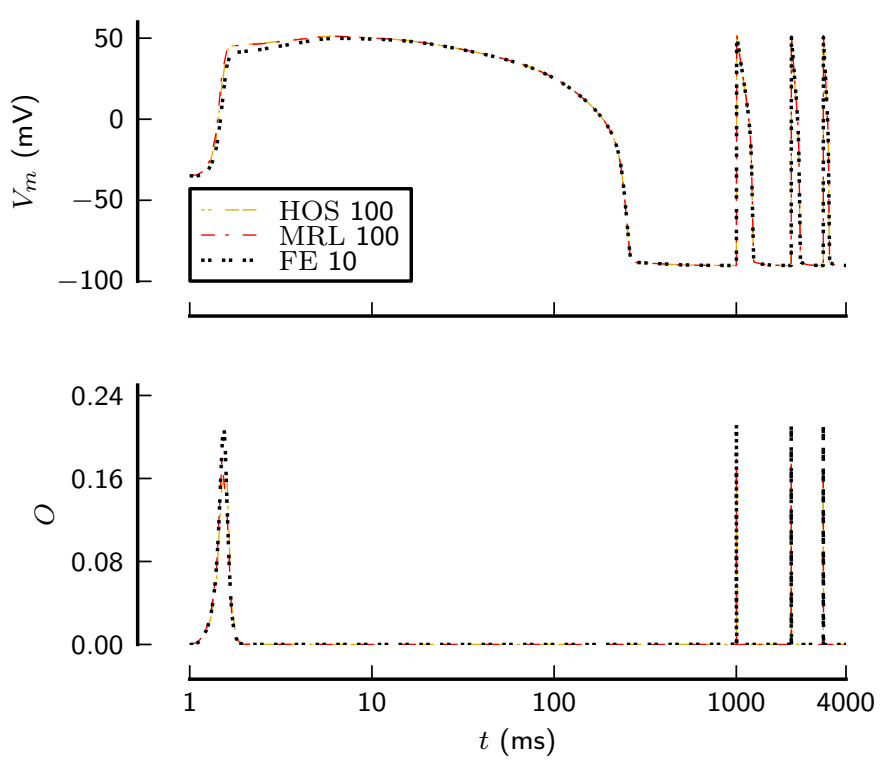

Fig. 5. Approximation properties of $\operatorname{HOS}(\Delta t=100 \mu \mathrm{s})$ and $\operatorname{MRL}(\Delta t=100 \mu \mathrm{s})$ compared to $\mathrm{FE}(\Delta t=10 \mu \mathrm{s})$ during a single cell simulation of 4 action potentials with cycle length (CL) of $1000 \mathrm{~ms}$ (logarithmic scale): membrane potential $V_{m}$ (top panel), open state occupancy $O$ (bottom panel).

\section{ACKNOWLEDGMENT}

During this study, TS was funded by University of Exeter PhD Studentship and VNB was partly supported by EPSRC grant EP/I029664/1. We are grateful to C.E. Clancy for the permission to use the original authors code for this study and S. Sherwin for encouraging discussions.

\section{REFERENCES}

[1] A. L. Hodgkin and A. F. Huxley, "A quantitative description of membrane current and its application to conduction and excitation in nerve," J. Physiol, vol. 117 , no. 4 , pp. 500-544, 1952.

[2] R. L. Burden and J. D. Faires, Numerical Analysis, 9th ed. Boston: Brooks/Cole, 2011.

[3] S. Rush and H. Larsen, "A practical algorithm for solving dynamic membrane equations," IEEE Trans. Biomed. Eng., vol. 25, no. 4, pp. 389-392, 1978.

[4] J. Sundnes, R. Artebrant, O. Skavhaug, and A. Tveito, "A second-order algorithm for solving dynamic cell membrane equations," IEEE Trans. Biomed. Eng., vol. 56, no. 10, pp. 2546-2548, 2009.

[5] M. Perego and A. Veneziani, "An efficient generalization of the Rush-Larsen method for solving electro-physiology membrane equations," Electronic Transactions on Numerical Analysis, vol. 35, pp. 234-256, 2009.

[6] M. E. Marsh, S. T. Ziaratgahi, and R. J. Spiteri, "The secrets to the success of the Rush-Larsen method and its generalizations," IEEE Trans. Biomed. Eng., vol. 59, no. 9, pp. 2506-2515, 2012.

[7] A. Reidman and K. Trivedi, "Numerical transient analysis of Markov models," Comput. Opns Res., vol. 15, no. 1, pp. 19-36, 1988.

[8] C. E. Clancy and Y. Rudy, "Na+ channel mutation that causes both Brugada and long-QT syndrome phenotypes: a simulation study of mechanism," Circulation, vol. 105, no. 10, pp. 1208-1213, 2002.

[9] C. H. Luo and Y. Rudy, "A dynamic-model of the cardiac ventricular action-potential. 1. Simulations of ionic currents and concentration changes," Circulation Research, vol. 74, no. 6, pp. 1071-1096, 1994.

[10] J. L. Zeng, K. R. Laurita, D. S. Rosenbaum, and Y. Rudy, "Two components of the delayed rectifier $\mathrm{K}^{+}$current in ventricular myocytes of the guinea-pig type. theoretical formulation and their role in repolarization," Circulation Research, vol. 77, no. 1, pp. 140-152, 1995.

[11] P. C. Viswanathan, R. M. Shaw, and Y. Rudy, "Effects of $I_{K r}$ and $I_{K s}$ heterogeneity on action potential duration and its rate dependence. a simulation study," Circulation, vol. 99, no. 18, May 1999.

[12] W. Stein et al., Sage Mathematics Software (Version 5.9), The Sage Development Team, 2013, http://www.sagemath.org. 


\title{
Supplementary material: \\ Exponential integrators for a Markov chain model of the fast sodium channel of cardiocytes
}

\author{
Tomáš Starý, Vadim N. Biktashev
}

The numeration of equations in this document continues from the main text, and the literature references are to the literature list in the main text, repeated in the end of this document for the reader's convenience.

\section{Cell Model Definition}

This section contains the definition of the model according to the authors' code [8]. The format of equations and subsections aims to correspond to the papers where those equations were published to facilitate a straightforward comparison. The known differences with the papers are marked by the sign: \#. Voltages are measured in $\mathrm{mV}$, time in ms and concentrations in $\mathrm{mmol} / \mathrm{L}$. The membrane currents are adjusted to the specific membrane capacitance $C=1 \mu \mathrm{F} / \mathrm{cm}^{2}[9]$ and are measured in $\mu \mathrm{A} / \mu \mathrm{F}$.

Standard ionic concentrations

$$
\begin{aligned}
{\left[\mathrm{Na}^{+}\right]_{o} } & =140 \quad \# \\
{\left[\mathrm{~K}^{+}\right]_{o} } & =4.5 \quad \# \\
{\left[\mathrm{Ca}^{2+}\right]_{o} } & =1.8
\end{aligned}
$$

which differs from [9] where $\left[\mathrm{Na}^{+}\right]_{o}=150 ;\left[\mathrm{K}^{+}\right]_{o}=5.4$.

Initial Values of Variables

$$
\begin{aligned}
x_{s 1} & =0 \quad \# \\
x_{s 2} & =0 \quad \# \\
V_{m} & =-95 \\
{\left[\mathrm{Ca}^{2+}\right]_{\mathrm{NSR}} } & =1.8 \\
{\left[\mathrm{Ca}^{2+}\right]_{\mathrm{JSR}} } & =1.8 \\
{\left[\mathrm{Ca}^{2+}\right]_{i} } & =0.00012 \\
b & =0.00141379 \\
g & =0.98831 \\
d & =6.17507 \times 10^{-6} \\
f & =0.999357 \\
X_{r} & =2.14606 \times 10^{-4} \\
{\left[\mathrm{Na}^{+}\right]_{i} } & =7.9 \quad \# \\
{\left[\mathrm{~K}^{+}\right]_{i} } & =147.23 \quad \#
\end{aligned}
$$

which differs from [9] where $\left[\mathrm{K}^{+}\right]_{i}=145 ;\left[\mathrm{Na}^{+}\right]_{i}=10$, and no initial values were given for $x_{s 1}$ and $x_{s 2}$.

Physical Constants

$$
\begin{aligned}
R & =8314 \\
F & =96485 \\
T & =310
\end{aligned}
$$


Cell geometry

$$
\begin{aligned}
L & =0.01 \\
r & =0.0011 \\
V_{\text {cell }} & =3.801 \times 10^{-5} \\
A_{\text {Geo }} & =2 \pi r^{2}+2 \pi r L \\
A_{\text {Cap }} & =2 A_{\text {Geo }} \\
V_{\text {myo }} & =2.58468 \times 10^{-5} \\
V_{\text {NSR }} & =0.0552 V_{\text {cell }} \\
V_{\text {JSR }} & =0.0048 V_{\text {cell }}
\end{aligned}
$$

$\mathrm{Na}^{+}-\mathrm{K}^{+}$pump : $I_{\mathrm{NaK}}$

$$
\begin{aligned}
I_{\mathrm{NaK}} & =1.5 f_{\mathrm{NaK}} \frac{1}{1+\left(10 /\left[\mathrm{Na}^{+}\right]_{i}\right)^{1.5}} \cdot \frac{\left[\mathrm{K}^{+}\right]_{o}}{\left[\mathrm{~K}^{+}\right]_{o}+1.5} \\
f_{\mathrm{NaK}} & =\frac{1}{1+0.1245 \exp \left(-0.1 \cdot \frac{V_{m} F}{R T}\right)+0.0365 \sigma \exp \left(\left(-V_{m} F\right) /(R T)\right)} \\
\sigma & =\frac{1}{7} \exp \left(\frac{\left[\mathrm{Na}^{+}\right]_{o}}{67.3}\right)-1
\end{aligned}
$$

which is identical to [9]

$I_{\mathrm{K} s}$, the Slow Component of the Delayed Rectifier $\mathrm{K}^{+}$Current

$$
\begin{aligned}
I_{\mathrm{K} s} & =\bar{G}_{\mathrm{K} s} x_{s 1} x_{s 2}\left(V_{m}-E_{\mathrm{Ks}}\right) \\
E_{\mathrm{K} s} & =(R T / F) \log \left(\left(4.5+P_{\mathrm{NaK}} 150\right) /\left(\left[\mathrm{K}^{+}\right]_{i}+P_{\mathrm{NaK}}\left[\mathrm{Na}^{+}\right]_{o}\right)\right) \quad \# \\
P_{\mathrm{NaK}} & =0.01833 \\
\bar{G}_{\mathrm{K} s} & =\left(0.433\left(1+0.6 /\left(1+\left(0.000038 /\left[\mathrm{Ca}^{2+}\right]_{i}\right)^{1.4}\right)\right)\right) \cdot 0.615 \quad \# \\
x_{s 1 \infty} & =1 /\left(1+\exp \left(-\left(V_{m}-1.5\right) / 16.7\right)\right) \\
x_{s 2 \infty} & =x_{s 1 \infty} \\
\tau_{x s 1} & =\left(0.0000719 \frac{V_{m}+30}{1-\exp \left(-0.148\left(V_{m}+30\right)\right)}+0.000131 \frac{V_{m}+30}{\exp \left(0.0687\left(V_{m}+30\right)\right)-1}\right)^{-1} \\
\tau_{x s 2} & =4 \tau_{x s 1}
\end{aligned}
$$

The definition of $E_{\mathrm{Ks}}$ in equation (51) differs from [11] by the hard-coded term for the $\left[\mathrm{K}^{+}\right]_{o}=4.5$ and $\left[\mathrm{Na}^{+}\right]_{o}=150$ rather than values defined by equations $(20,21)$ where $\left[\mathrm{K}^{+}\right]_{o}=4.5$ and $\left[\mathrm{Na}^{+}\right]_{o}=140$ are parameters.

The definition of $\bar{G}_{\mathrm{K} s}$ in equation (53) is multiplied by 0.615 "to simulate the intramural heterogeneity", which is slightly different from the factor 0.652 used in Viswanathan et al. (1999) [11] to simulate epicardial cell.

Otherwise the $I_{\mathrm{Ks}}$ definition is identical to [11].

$$
\begin{aligned}
\frac{\mathrm{d} x_{s 1}}{\mathrm{~d} t} & =\frac{x_{s 1 \infty}-x_{s 1}}{\tau_{x s 1}} \\
\frac{\mathrm{d} x_{s 2}}{\mathrm{~d} t} & =\frac{x_{s 2 \infty}-x_{s 2}}{\tau_{x s 2}}
\end{aligned}
$$

$I_{\mathrm{K} r}$, the Fast Component of the Delayed Rectifier $\mathrm{K}^{+}$Current

$$
\begin{aligned}
I_{\mathrm{K} r} & =\bar{G}_{\mathrm{K} r} X_{r} R_{\mathrm{K} r}\left(V_{m}-E_{\mathrm{K} r}\right) \\
\bar{G}_{\mathrm{K} r} & =0.02614 \sqrt{\left[\mathrm{K}^{+}\right]_{o} / 5.4} \\
X_{r \infty} & =1 /\left(1+\exp \left(-\left(V_{m}+21.5\right) / 7.5\right)\right)
\end{aligned}
$$




$$
\begin{aligned}
R_{\mathrm{K} r} & =1 /\left(1+\exp \left(\left(V_{m}+9\right) / 22.4\right)\right) \\
E_{\mathrm{K} r} & =((R T) / F) \log \left(\left[\mathrm{K}^{+}\right]_{o} /\left[\mathrm{K}^{+}\right]_{i}\right) \\
\tau_{x r} & =\left(0.00138 \frac{V_{m}+14.2}{1-\exp \left(-0.123\left(V_{m}+14.2\right)\right)}+0.00061 \frac{V_{m}+38.9}{\exp \left(0.145\left(V_{m}+38.9\right)\right)-1}\right)^{-1}
\end{aligned}
$$

which is identical to [10]. The original notation for $R_{\mathrm{K} r}$ was $R$; we use $R$ for the gas constant in this section, and for one of the state occupancies of the Markov Chain model elsewhere in the rest of the paper.

$$
\frac{\mathrm{d} X_{r}}{\mathrm{~d} t}=\frac{X_{r \infty}-X_{r}}{\tau_{x r}}
$$

Time-independent $\mathrm{K}^{+}$current: $I_{\mathrm{K} 1}$

$$
\begin{aligned}
I_{\mathrm{K} 1} & =\bar{G}_{\mathrm{K} 1} \mathrm{~K} 1_{\infty}\left(V_{m}-E_{\mathrm{K} 1}\right) \\
E_{\mathrm{K} 1} & =(R T / F) \log \left(\left[\mathrm{K}^{+}\right]_{o} /\left[\mathrm{K}^{+}\right]_{i}\right) \\
\bar{G}_{\mathrm{K} 1} & =0.75 \cdot \sqrt{\left(\left[\mathrm{K}^{+}\right]_{o} / 5.4\right)} \\
\alpha_{\mathrm{K} 1} & =1.02 /\left(1+\exp \left(0.2385\left(V_{m}-E_{\mathrm{K} 1}-59.215\right)\right)\right) \\
\beta_{\mathrm{K} 1} & =\frac{0.49124 \exp \left(0.08032\left(V_{m}-E_{\mathrm{K} 1}+5.476\right)\right)+\exp \left(0.06175\left(V_{m}-E_{\mathrm{K} 1}-594.31\right)\right)}{1+\exp \left(-0.5143\left(V_{m}-E_{\mathrm{K} 1}+4.753\right)\right)}
\end{aligned}
$$

which is identical to [9].

$$
\mathrm{K} 1_{\infty}=\alpha_{\mathrm{K} 1} /\left(\alpha_{\mathrm{K} 1}+\beta_{\mathrm{K} 1}\right)
$$

Plateau $\mathrm{K}^{+}$current: $I_{\mathrm{K} p}$

$$
\begin{aligned}
I_{\mathrm{K} p} & =0.00552 K_{p}\left(V_{m}-E_{\mathrm{K} 1}\right) \\
K_{p} & =1 /\left(1+\exp \left(\left(7.488-V_{m}\right) / 5.98\right)\right)
\end{aligned}
$$

equivalent to [9] with an update from [10].

$$
I_{\mathrm{K}}=I_{\mathrm{K} 1}+I_{\mathrm{K} p}
$$

Currents through the L-type $\mathrm{Ca}^{+2}$ channel $I_{\mathrm{Ca} L}$

$$
\begin{aligned}
I_{\mathrm{Ca} L} & =I_{\mathrm{Ca}}+I_{\mathrm{CaK}}+I_{\mathrm{CaNa}} \\
I_{\mathrm{Ca}} & =d f f_{\mathrm{Ca}} \bar{I}_{\mathrm{Ca}} \\
I_{\mathrm{CaK}} & =d f f_{\mathrm{Ca}} \bar{I}_{\mathrm{CaK}} \\
I_{\mathrm{CaNa}} & =d f f_{\mathrm{Ca}} \bar{I}_{\mathrm{CaNa}} \\
\bar{I}_{\mathrm{Ca}} & =P_{\mathrm{Ca}} z_{\mathrm{Ca}}^{2} \frac{\left(V_{m} F^{2}\right)}{R T} \frac{\gamma_{\mathrm{Ca} i}\left[\mathrm{Ca}^{2+}\right]_{i} \exp \left(\left(z_{\mathrm{Ca}} V_{m} F\right) /(R T)\right)-\gamma_{\mathrm{Cao}}\left[\mathrm{Ca}^{2+}\right]_{o}}{\exp \left(\left(z_{\mathrm{Ca}} V_{m} F\right) /(R T)\right)-1} \\
\bar{I}_{\mathrm{CaNa}} & =P_{\mathrm{Na}} z_{\mathrm{Na}}^{2} \frac{\left(V_{m} F^{2}\right)}{R T} \frac{\gamma_{\mathrm{Na} i}\left[\mathrm{Na}^{+}\right]_{i} \exp \left(\left(z_{\mathrm{Na}} V_{m} F\right) /(R T)\right)-\gamma_{\mathrm{Nao}}\left[\mathrm{Na}^{+}\right]_{o}}{\exp \left(\left(z_{\mathrm{Na}} V_{m} F\right) /(R T)\right)-1} \\
\bar{I}_{\mathrm{CaK}} & =P_{\mathrm{K}} z_{\mathrm{K}}^{2} \frac{\left(V_{m} F^{2}\right)}{R T} \frac{\gamma_{\mathrm{K} i}\left[\mathrm{~K}^{+}\right]_{i} \exp \left(\left(z_{\mathrm{K}} V_{m} F\right) /(R T)\right)-\gamma_{\mathrm{Ko}}\left[\mathrm{K}^{+}\right]_{o}}{\exp \left(\left(z_{\mathrm{K}} V_{m} F\right) /(R T)\right)-1} \\
P_{\mathrm{Ca}} & =5.4 \times 10^{-4} \quad \gamma_{\mathrm{Ca} i}=1 \quad \gamma_{\mathrm{Na} i}=0.341 \\
P_{\mathrm{Na}} & =6.75 \times 10^{-7}=0.75 \\
P_{\mathrm{K}} & =1.93 \times 10^{-7} \quad \gamma_{\mathrm{K} i}=0.75 \quad \gamma_{\mathrm{Ko}}=0.75 \\
f_{\mathrm{Ca}} & =1 /\left(1+\left[\mathrm{Ca}^{2+}\right]_{i} / K_{m \mathrm{Ca}}\right) \\
K_{m \mathrm{Ca}} & =0.0006
\end{aligned}
$$




$$
\begin{aligned}
d_{\infty} & =1 /\left(1+\exp \left(-\left(V_{m}+10\right) / 6.24\right)\right) \\
\tau_{d} & =d_{\infty}\left(1-\exp \left(-\left(V_{m}+10\right) / 6.24\right)\right) /\left(0.035\left(V_{m}+10\right)\right) \\
f_{\infty} & =\left(1 /\left(1+\exp \left(\left(V_{m}+32\right) / 8\right)\right)\right)+\left(0.6 /\left(1+\exp \left(\left(50-V_{m}\right) / 20\right)\right)\right) \\
\tau_{f} & =1 /\left(0.0197 \exp \left(-\left(0.0337\left(V_{m}+10\right)^{2}\right)\right)+0.02\right)
\end{aligned}
$$

Equation (91) differs from [9] which has 8.6 rather than 8 in the denominator of the argument of the first exponential. Otherwise, these equations are exactly the same as in [9].

$$
\begin{aligned}
z_{\mathrm{Na}} & =1 \\
z_{\mathrm{K}} & =1 \\
z_{\mathrm{Ca}} & =2 \\
\frac{\mathrm{d} d}{\mathrm{~d} t} & =\frac{d_{\infty}-d}{\tau_{d}} \\
\frac{\mathrm{d} f}{\mathrm{~d} t} & =\frac{f_{\infty}-f}{\tau_{f}}
\end{aligned}
$$

$\mathrm{Ca}^{2+}$ Current Through T-Type $\mathrm{Ca}^{2+}$ Channels $I_{\mathrm{Ca}(T)}$

$$
\begin{aligned}
I_{\mathrm{Ca}(T)} & =\bar{G}_{\mathrm{Ca}(T)} b^{2} g\left(V_{m}-E_{\mathrm{Ca}}\right) \\
\bar{G}_{\mathrm{Ca}(T)} & =0.05 \\
b_{\infty} & =1 /\left(1+\exp \left(-\left(V_{m}+14\right) / 10.8\right)\right) \\
g_{\infty} & =1 /\left(1+\exp \left(\left(V_{m}+60\right) / 5.6\right)\right) \\
E_{\mathrm{Ca}} & =(R T /(2 F)) \log \left(\left[\mathrm{Ca}^{2+}\right]_{o} /\left[\mathrm{Ca}^{2+}\right]_{i}\right) \\
\tau_{b} & =3.7+6.1 /\left(1+\exp \left(\left(V_{m}+25\right) / 4.5\right)\right) \\
\tau_{g} & =-0.875 V_{m}+12 \text { for: } V_{m} \leq 0 ; \text { and } \tau_{g}=12 \text { for: } V_{m}>0
\end{aligned}
$$

which correspond exactly to [10].

$$
\begin{aligned}
& \frac{\mathrm{d} b}{\mathrm{~d} t}=\frac{b_{\infty}-b}{\tau_{b}} \\
& \frac{\mathrm{d} g}{\mathrm{~d} t}=\frac{g_{\infty}-g}{\tau_{g}}
\end{aligned}
$$

$\mathrm{Na}^{+}-\mathrm{Ca}^{+}$exchanger: $I_{\mathrm{NaCa}}$

$$
\begin{aligned}
I_{\mathrm{NaCa}} & =\frac{2.5 \times 10^{-4} \exp \left((\eta-1) V_{m} \frac{F}{R T}\right)\left(\exp \left(V_{m} \frac{F}{R T}\right)\left[\mathrm{Na}^{+}\right]_{i}^{3}\left[\mathrm{Ca}^{2+}\right]_{o}-\left[\mathrm{Na}^{+}\right]_{o}^{3}\left[\mathrm{Ca}^{2+}\right]_{i}\right)}{1+1 \times 10^{-4} \exp \left((\eta-1) V_{m} \frac{F}{R T}\right)\left(\exp \left(V_{m} \frac{F}{R T}\right)\left[\mathrm{Na}^{+}\right]_{i}^{3}\left[\mathrm{Ca}^{2+}\right]_{o}+\left[\mathrm{Na}^{+}\right]_{o}^{3}\left[\mathrm{Ca}^{2+}\right]_{i}\right)} \quad \# \\
\eta & =0.15 \#
\end{aligned}
$$

Here $I_{\mathrm{NaCa}}$ depends on external $\left[\mathrm{Ca}^{2+}\right]_{o},\left[\mathrm{Na}^{+}\right]_{o}$ as well as internal $\left[\mathrm{Na}^{+}\right]_{i},\left[\mathrm{Ca}^{2+}\right]_{i}$ concentrations, which is different from $[9]$ where it depended only on external concentrations $\left[\mathrm{Ca}^{2+}\right]_{o},\left[\mathrm{Na}^{+}\right]_{o}$. The variable $\eta=0.35$ in [9].

Nonspecific $\mathrm{Ca}^{2+}$-activated current: $I_{n s(\mathrm{Ca})}$

$$
\begin{aligned}
\bar{I}_{n s \mathrm{~K}} & =1.75 \times 10^{-7} \frac{V_{m} F^{2}}{R T} \cdot \frac{0.75\left[\mathrm{~K}^{+}\right]_{i} \exp \left(\left(V_{m} F\right) /(R T)\right)-0.75\left[\mathrm{~K}^{+}\right]_{o}}{\exp \left(V_{m} F /(R T)\right)-1} \\
I_{n s \mathrm{~K}} & =\bar{I}_{n s \mathrm{~K}} \frac{1}{1+\left(0.0012 /\left[\mathrm{Ca}^{2+}\right]_{i}\right)^{3}} \\
\bar{I}_{n s \mathrm{Na}} & =1.75 \times 10^{-7} \frac{V_{m} F^{2}}{R T} \cdot \frac{0.75\left[\mathrm{Na}^{+}\right]_{i} \exp \left(\left(V_{m} F\right) /(R T)\right)-0.75\left[\mathrm{Na}^{+}\right]_{o}}{\exp \left(V_{m} F /(R T)\right)-1} \\
I_{n s \mathrm{Na}} & =\bar{I}_{n s \mathrm{Na}} \frac{1}{1+\left(0.0012 /\left[\mathrm{Ca}^{2+}\right]_{i}\right)^{3}} \\
I_{n s(\mathrm{Ca})} & =I_{n s \mathrm{~K}}+I_{n s \mathrm{Na}} \\
P_{n s(\mathrm{Ca})} & =1.75 \times 10^{-7}
\end{aligned}
$$

This is almost identical to [9] except the latter also made a definition for $E_{n s(\mathrm{Ca})}$ which however was not used. 
Sarcolemmal $\mathrm{Ca}^{+2}$ pump: $I_{p(\mathrm{Ca})}$

$$
I_{p(\mathrm{Ca})}=1.15 \frac{\left[\mathrm{Ca}^{2+}\right]_{i}}{0.0005+\left[\mathrm{Ca}^{2+}\right]_{i}}
$$

identical to [9].

$\mathrm{Ca}^{+2}$ background current: $I_{\mathrm{Ca} b}$

$$
\begin{aligned}
I_{\mathrm{Ca} b} & =0.003016\left(V_{m}-E_{\mathrm{Ca}}\right) \\
E_{\mathrm{Ca}} & =R T /(2 F) \log \left(\left[\mathrm{Ca}^{2+}\right]_{o} /\left[\mathrm{Ca}^{2+}\right]_{i}\right)
\end{aligned}
$$

identical to [9].

$\mathrm{Na}^{+}$background current: $I_{\mathrm{Na} b}$

$$
\begin{aligned}
E_{\mathrm{Na}} & =((R T) / F) \log \left(\left[\mathrm{Na}^{+}\right]_{o} /\left[\mathrm{Na}^{+}\right]_{i}\right) \\
I_{\mathrm{Na} b} & =0.00141\left(V_{m}-E_{\mathrm{Na}}\right)
\end{aligned}
$$

identical to [9].

$\mathrm{Ca}^{2+}$ uptake and leakage of NSR: $I_{u p}$ and $I_{\text {leak }}$

$$
\begin{aligned}
I_{\text {up }} & =0.00875\left[\mathrm{Ca}^{2+}\right]_{i} /\left(\left[\mathrm{Ca}^{2+}\right]_{i}+0.00092\right) \quad \# \\
K_{\text {leak }} & =0.005 / 15 \\
I_{\text {leak }} & =K_{\text {leak }}\left[\mathrm{Ca}^{2+}\right]_{\mathrm{NSR}}
\end{aligned}
$$

The definition of $I_{u p}$ in [9] is ambiguous. This version is consistent with one possible understanding.

$$
\mathrm{Ca}^{+2} \text { Fluxes in NSR }
$$

$$
\frac{\mathrm{d}\left[\mathrm{Ca}^{2+}\right]_{\mathrm{NSR}}}{\mathrm{d} t}=\left(I_{u p}-I_{\text {leak }}-I_{t r} V_{\mathrm{JSR}} / V_{\mathrm{NSR}}\right)
$$

$\mathrm{Ca}^{2+}$ Fluxes in Myoplasm

$$
\begin{aligned}
I_{t \mathrm{Ca}} & =I_{\mathrm{Ca}}+I_{\mathrm{Ca} b}+I_{p(\mathrm{Ca})}-2 I_{\mathrm{NaCa}}+I_{\mathrm{Ca}(T)} \\
\Delta\left[\mathrm{Ca}^{2+}\right]_{i} & =-\Delta t\left(\left(\left(I_{t \mathrm{Ca}} A_{\mathrm{Cap}}\right) /\left(V_{\text {myo }} 2 F\right)\right)+\left(\left(I_{\text {up }}-I_{\text {leak }}\right) V_{\mathrm{NSR}} / V_{\text {myo }}\right)-\left(I_{\text {rel }} V_{\mathrm{JSR}} / V_{\text {myo }}\right)\right) \\
{\left[\mathrm{Ca}^{2+}\right]_{i o n} } & =\mathrm{TRPN}+\mathrm{CMDN}+\Delta\left[\mathrm{Ca}^{2+}\right]_{i}+\left[\mathrm{Ca}^{2+}\right]_{i} \\
B & =0.05+0.07-\left[\mathrm{Ca}^{2+}\right]_{i o n}+0.0005+0.00238 \\
C & =(0.00238 \cdot 0.0005)-\left(\left[\mathrm{Ca}^{2+}\right]_{i o n}(0.0005+0.00238)\right)+(0.07 \cdot 0.00238)+(0.05 \cdot 0.0005) \\
D & =-0.0005 \cdot 0.00238\left[\mathrm{Ca}^{2+}\right]_{i o n} \\
F_{a b} & =\sqrt{\left(B^{2}-3 C\right)} \\
{\left[\mathrm{Ca}^{2+}\right]_{i} } & =1.5 F_{a b} \cos \left(\arccos \left(\left(9 B C-2 B^{3}-27 D\right) /\left(2\left(B^{2}-3 C\right)^{1.5}\right)\right) / 3\right)-(B / 3)
\end{aligned}
$$

This definition merely summarises computations that are done in the code, which de facto describe a time-stepping algorithm for a system of a differential equation and a finite constraint, rather than the equation and the constraint themselves, hence the time step $\Delta t$ is present in (125). Any attempts of higher-order numerical approximations would have to take this into account. The explicit solution of the finite constraint given by the cubic formula (131) follows [10] whereas [9] used Steffensen's iterations for that purpose. 
$\mathrm{Ca}^{2+}$ Fluxes in JSR

$$
\begin{aligned}
\Delta\left[\mathrm{Ca}^{2+}\right]_{\mathrm{JSR}} & =\Delta t\left(I_{\text {tr }}-I_{\text {rel }}\right) \\
b_{\mathrm{JSR}} & =10-\mathrm{CSQN}-\Delta\left[\mathrm{Ca}^{2+}\right]_{\mathrm{JSR}}-\left[\mathrm{Ca}^{2+}\right]_{\mathrm{JSR}}+0.8 \\
c_{\mathrm{JSR}} & =0.8\left(\mathrm{CSQN}+\Delta\left[\mathrm{Ca}^{2+}\right]_{\mathrm{JSR}}+\left[\mathrm{Ca}^{2+}\right]_{\mathrm{JSR}}\right) \\
{\left[\mathrm{Ca}^{2+}\right]_{\mathrm{JSR}} } & =\left(\sqrt{\left(b_{\mathrm{JSR}}^{2}+4 c_{\mathrm{JSR}}\right)}-b_{\mathrm{JSR}}\right) / 2
\end{aligned}
$$

Ditto: $\Delta t$ is present in (132).

Sodium Ion Fluxes

$$
\begin{aligned}
I_{t \mathrm{Na}} & =I_{\mathrm{Na}}+I_{\mathrm{Na} b}+I_{\mathrm{CaNa}}+I_{n s \mathrm{Na}}+3 I_{\mathrm{NaK}}+3 I_{\mathrm{NaCa}} \\
\frac{\mathrm{d}\left[\mathrm{Na}^{+}\right]_{i}}{\mathrm{~d} t} & =-\left(I_{t \mathrm{Na}} A_{C a p}\right) /\left(V_{m y o} F\right)
\end{aligned}
$$

$\left[\mathrm{Na}^{+}\right]_{i}$ is constant in [9], [10], [11].

Potassium Ion Fluxes

$$
\begin{aligned}
I_{t \mathrm{~K}} & =I_{\mathrm{K} r}+I_{\mathrm{K} s}+I_{\mathrm{K}}+I_{\mathrm{CaK}}+I_{n s \mathrm{~K}}-2 I_{\mathrm{NaK}}+I_{t o}+I_{s t} \\
\frac{\mathrm{d}\left[\mathrm{K}^{+}\right]_{i}}{\mathrm{~d} t} & =-\left(I_{t \mathrm{~K}} A_{C a p}\right) /\left(V_{m y o} F\right)
\end{aligned}
$$

$\left[\mathrm{K}^{+}\right]_{i}$ is constant in [9], [10], [11].

$\mathrm{Ca}^{2+}$ buffers in the myoplasm

$$
\begin{aligned}
\operatorname{TRPN} & =0.07\left[\mathrm{Ca}^{2+}\right]_{i} /\left(\left[\mathrm{Ca}^{2+}\right]_{i}+0.0005\right) \\
\mathrm{CMDN} & =0.05\left[\mathrm{Ca}^{2+}\right]_{i} /\left(\left[\mathrm{Ca}^{2+}\right]_{i}+0.00238\right)
\end{aligned}
$$

identical to [9].

$\mathrm{Ca}^{2+}$ buffer in JSR and SCQN

$$
\mathrm{CSQN}=10\left(\left[\mathrm{Ca}^{2+}\right]_{\mathrm{JSR}} /\left(\left[\mathrm{Ca}^{2+}\right]_{\mathrm{JSR}}+0.8\right)\right)
$$

identical to [9].

CICR From Junctional SR (JSR)

$$
\begin{aligned}
I_{\text {rel }} & =G_{\text {rel }} \mathrm{ryr}_{\text {open }} \mathrm{ryr}_{\text {close }}\left(\left[\mathrm{Ca}^{2+}\right]_{\mathrm{JSR}}-\left[\mathrm{Ca}^{2+}\right]_{i}\right) \\
G_{\text {rel }} & =150 /\left(1+\exp \left(I_{t \mathrm{Ca}}+5\right) / 0.9\right) \quad \# \\
\mathrm{ryr}_{\text {open }} & =1 /\left(1+\exp \left(\left(-t_{c}+4\right) / 0.5\right)\right) \quad \# \\
\operatorname{ryr}_{\text {close }} & =1-\left(1 /\left(1+\exp \left(\left(-t_{c}+4\right) / 0.5\right)\right)\right) \quad \#
\end{aligned}
$$

Here is another deviation of the model description from the standard form of a system of ODEs, and this also would have to be taken into account in any attempts of higher-order schemes. Variables ryr close $_{\text {and }}$ ryr $_{\text {open }}=1-\mathrm{ryr}_{\text {close }}$ ensure that the calcium release channel is open at a fuzzy time interval around $4 \mathrm{~ms}$ after the steepest point of the upstroke of the action potential. This is done using an additional time variable $t_{c}$ which is linked to the $t$, that is $\mathrm{d} t_{c} / \mathrm{d} t=1 \mathrm{most}$ of the time, except $t_{c}$ is reset to zero each time the $\frac{\mathrm{d} V_{m}}{\mathrm{~d} t}$ reaches a significant local maximum, "significant" meaning $\frac{\mathrm{d} V_{m}}{\mathrm{~d} t}>1 \mathrm{mV} / \mathrm{ms}$. In [9], $G_{r e l}$ is defined differently from (145) and calcium release proceeds with a different dynamics from (146,147), e.g. it starts sharply $2 \mathrm{~ms}$ after the the time of the maximum $\frac{\mathrm{d} V_{m}}{\mathrm{~d} t}$. 
Translocation of $\mathrm{Ca}^{2+}$ ions from NSR to JSR: $I_{t r}$

$$
I_{t r}=\left(\left[\mathrm{Ca}^{2+}\right]_{\mathrm{NSR}}-\left[\mathrm{Ca}^{2+}\right]_{\mathrm{JSR}}\right) / 180
$$

identical to [9].

Total time-independent current: $I_{v}$

$$
I_{v}=I_{\mathrm{Na} b}+I_{\mathrm{NaK}}+I_{p(\mathrm{Ca})}+I_{\mathrm{K} p}+I_{\mathrm{Ca} b}+I_{\mathrm{K} 1}
$$

identical to [9].

Total Current

$$
\begin{aligned}
I_{t}= & I_{\mathrm{K} r}+I_{\mathrm{K} s}+I_{\mathrm{K}}+I_{\mathrm{CaK}}+I_{n s \mathrm{~K}}-2 I_{\mathrm{NaK}}+I_{\mathrm{Na}}+I_{\mathrm{Na} b}+I_{\mathrm{CaNa}}+I_{n s \mathrm{Na}}+3 I_{\mathrm{NaK}}+ \\
& 3 I_{\mathrm{NaCa}}+I_{\mathrm{Ca}}+I_{\mathrm{Ca} b}+I_{p(\mathrm{Ca})}-2 I_{\mathrm{NaCa}}+I_{\mathrm{Ca}(T)}
\end{aligned}
$$

\section{Membrane Potential}

$$
\frac{\mathrm{d} V_{m}}{\mathrm{~d} t}=-I_{t}
$$

\section{VI. $I_{\mathrm{Na}}$ MARKov Chain Model Definition}

Up to the choice of notation, we use the model described in [8]. The fast sodium current is defined by

$$
I_{\mathrm{Na}}=G_{\mathrm{Na}}\left(V_{m}-E_{\mathrm{Na}}\right) O,
$$

where the channel open probability $O$ is defined by the system of ODEs

$$
\begin{aligned}
\frac{\mathrm{d} O}{\mathrm{~d} t} & =\alpha_{P O} P+\alpha_{U O} U-\left(\alpha_{O P}+\alpha_{O U}\right) O \\
\frac{\mathrm{d} P}{\mathrm{~d} t} & =\alpha_{Q P} Q+\alpha_{U P} U+\alpha_{O P} O-\left(\alpha_{P Q}+\alpha_{P U}+\alpha_{P O}\right) P \\
\frac{\mathrm{d} Q}{\mathrm{~d} t} & =\alpha_{R Q} R+\alpha_{T Q} T+\alpha_{P Q} P-\left(\alpha_{Q R}+\alpha_{Q T}+\alpha_{Q P}\right) Q \\
\frac{\mathrm{d} R}{\mathrm{~d} t} & =\alpha_{S R} S+\alpha_{Q R} Q-\left(\alpha_{R S}+\alpha_{R Q}\right) R \\
\frac{\mathrm{d} S}{\mathrm{~d} t} & =\alpha_{T S} T+\alpha_{R S} R-\left(\alpha_{S T}+\alpha_{S R}\right) S \\
\frac{\mathrm{d} T}{\mathrm{~d} t} & =\alpha_{Q T} Q+\alpha_{S T} S+\alpha_{U T} U-\left(\alpha_{T Q}+\alpha_{T S}+\alpha_{T U}\right) T \\
\frac{\mathrm{d} U}{\mathrm{~d} t} & =\alpha_{T U} T+\alpha_{P U} P+\alpha_{V U} V+\alpha_{O U} O-\left(\alpha_{U T}+\alpha_{U P}+\alpha_{U O}+\alpha_{U V}\right) U \\
\frac{\mathrm{d} V}{\mathrm{~d} t} & =\alpha_{U V} U+\alpha_{W V} W-\left(\alpha_{V U}+\alpha_{V W}\right) V \\
\frac{\mathrm{d} W}{\mathrm{~d} t} & =\alpha_{V W} V-\alpha_{W V} W
\end{aligned}
$$

with the transition rates defined by

$$
\begin{aligned}
& \alpha_{R Q}= \alpha_{S T}=\alpha_{11}=\frac{3.802}{0.1027 \mathrm{e}^{-V_{m} / 17.0}+0.20 \mathrm{e}^{-V_{m} / 150}} \\
& \alpha_{Q P}= \alpha_{T U}=\alpha_{12}=\frac{3.802}{0.1027 \mathrm{e}^{-V_{m} / 15.0}+0.23 \mathrm{e}^{-V_{m} / 150}} \\
& \alpha_{P O}=\alpha_{13}=\frac{3.802}{0.1027 \mathrm{e}^{-V_{m} / 12.0}+0.25 \mathrm{e}^{-V_{m} / 150}} \\
& \alpha_{Q R}=\alpha_{T S}=\beta_{11}=0.1917 \mathrm{e}^{-V_{m} / 20.3} \\
& \alpha_{P Q}=\alpha_{U T}=\beta_{12}=0.20 \mathrm{e}^{-\left(V_{m}-5\right) / 20.3}
\end{aligned}
$$




$$
\begin{gathered}
\alpha_{O P}=\beta_{13}=0.22 \mathrm{e}^{-\left(V_{m}-10\right) / 20.3} \\
\alpha_{U P}=\alpha_{T Q}=\alpha_{S R}=\alpha_{3}=3.7933 \cdot 10^{-7} \mathrm{e}^{-V_{m} / 7.7} \\
\alpha_{P U}=\alpha_{Q T}=\alpha_{R S}=\beta_{3}=8.4 \cdot 10^{-3}+2 \cdot 10^{-5} V_{m} \\
\alpha_{O U}=\alpha_{2}=9.178 \mathrm{e}^{V_{m} / 29.68} \\
\alpha_{U O}=\beta_{2}=\frac{\alpha_{13} \alpha_{2} \alpha_{3}}{\beta_{13} \beta_{3}} \\
\alpha_{U V}=\alpha_{4}=\alpha_{2} / 100 \\
\alpha_{V U}=\beta_{4}=\alpha_{3} \\
\alpha_{V W}=\alpha_{5}=\alpha_{2} /\left(9.5 \cdot 10^{4}\right) \\
\alpha_{W V}=\beta_{5}=\alpha_{3} / 50
\end{gathered}
$$

VII. Details OF THE HYBRID OPERATOR SPlitTING METHOD

\section{A. Operator splitting}

In the hybrid method we use operator splitting. The system of equations (153)-(161) is considered as an ODE

$$
\frac{\mathrm{d} \vec{u}}{\mathrm{~d} t}=\boldsymbol{A}\left(V_{m}(t)\right) \vec{u}
$$

for the vector-function $\vec{u}=(O, P, Q, R, S, T, U, V, W)^{\top}=\vec{u}(t)$, and the transition matrix is split into the sum

$$
\boldsymbol{A}=\boldsymbol{A}_{0}+\boldsymbol{A}_{1}+\boldsymbol{A}_{2}
$$

of the matrix $\boldsymbol{A}_{0}$ of transition rates that are fast at high values of $V_{m}$,

$$
\boldsymbol{A}_{0}=\left[\begin{array}{ccccccccc}
-\alpha_{O U} & \alpha_{P O} & 0 & 0 & 0 & 0 & 0 & 0 & 0 \\
0 & -\alpha_{P O} & \alpha_{Q P} & 0 & 0 & 0 & 0 & 0 & 0 \\
0 & 0 & -\alpha_{Q P} & \alpha_{R Q} & 0 & 0 & 0 & 0 & 0 \\
0 & 0 & 0 & -\alpha_{R Q} & 0 & 0 & 0 & 0 & 0 \\
0 & 0 & 0 & 0 & -\alpha_{S T} & 0 & 0 & 0 & 0 \\
0 & 0 & 0 & 0 & \alpha_{S T} & -\alpha_{T U} & 0 & 0 & 0 \\
\alpha_{O U} & 0 & 0 & 0 & 0 & \alpha_{T U} & 0 & 0 & 0 \\
0 & 0 & 0 & 0 & 0 & 0 & 0 & 0 & 0 \\
0 & 0 & 0 & 0 & 0 & 0 & 0 & 0 & 0
\end{array}\right]
$$

the matrix $\boldsymbol{A}_{1}$ of transition rates that are fast at low values of $V_{m}$,

$$
\boldsymbol{A}_{1}=\left[\begin{array}{ccccccccc}
-\alpha_{O P} & 0 & 0 & 0 & 0 & 0 & 0 & 0 & 0 \\
\alpha_{O P} & -\alpha_{P Q} & 0 & 0 & 0 & 0 & 0 & 0 & 0 \\
0 & \alpha_{P Q} & -\alpha_{Q R} & 0 & 0 & 0 & 0 & 0 & 0 \\
0 & 0 & \alpha_{Q R} & 0 & 0 & 0 & 0 & 0 & 0 \\
0 & 0 & 0 & 0 & 0 & \alpha_{T S} & 0 & 0 & 0 \\
0 & 0 & 0 & 0 & 0 & -\alpha_{T S} & \alpha_{U T} & 0 & 0 \\
0 & 0 & 0 & 0 & 0 & 0 & -\alpha_{U T} & 0 & 0 \\
0 & 0 & 0 & 0 & 0 & 0 & 0 & 0 & 0 \\
0 & 0 & 0 & 0 & 0 & 0 & 0 & 0 & 0
\end{array}\right]
$$

and the matrix $\boldsymbol{A}_{2}$ of uniformly slow transition rates,

$$
\boldsymbol{A}_{2}=\left[\begin{array}{ccccccccc}
0 & 0 & 0 & 0 & 0 & 0 & \alpha_{U O} & 0 & 0 \\
0 & -\alpha_{P U} & 0 & 0 & 0 & 0 & \alpha_{U P} & 0 & 0 \\
0 & 0 & -\alpha_{Q T} & 0 & 0 & \alpha_{T Q} & 0 & 0 & 0 \\
0 & 0 & 0 & -\alpha_{R S} & \alpha_{S R} & 0 & 0 & 0 & 0 \\
0 & 0 & 0 & \alpha_{R S} & -\alpha_{S R} & 0 & 0 & 0 & 0 \\
0 & 0 & \alpha_{Q T} & 0 & 0 & -\alpha_{T Q} & 0 & \alpha_{V U} & 0 \\
0 & \alpha_{P U} & 0 & 0 & 0 & 0 & -\left(\alpha_{U P}+\alpha_{U O}+\alpha_{U V}\right) & \left.\alpha_{U V}+\alpha_{V W}\right) & \alpha_{W V} \\
0 & 0 & 0 & 0 & 0 & 0 & 0 & \alpha_{V W} & -\alpha_{W V}
\end{array}\right] .
$$




\section{B. The substeps}

Every timestep is then done in three substeps, each using one of the three matrices $\boldsymbol{A}_{m}, m=0,1,2$ :

$$
\begin{aligned}
\vec{u}_{n+1 / 3} & =\exp \left(\Delta t \boldsymbol{A}_{0}\left(V_{m}\left(t_{n}\right)\right)\right) \vec{u}_{n}, \\
\vec{u}_{n+2 / 3} & =\exp \left(\Delta t \boldsymbol{A}_{1}\left(V_{m}\left(t_{n}\right)\right)\right) \vec{u}_{n+1 / 3}, \\
\vec{u}_{n+1} & =\vec{u}_{n+2 / 3}+\Delta t \boldsymbol{A}_{2}\left(V_{m}\left(t_{n}\right)\right) \vec{u}_{n+2 / 3} .
\end{aligned}
$$

Note that $V_{m}$ in all cases is evaluated at $t=t_{n}$, in which we simply follow the original Rush-Larsen idea of "freezing" $V_{m}$ for the duration of the time step. The matrix exponentials in (17) and (18) can be understood in terms of matrix Taylor series [7], or the product of the matrix exponential by the corresponding vector $\vec{u}$ can be understood just as the solutions of an initial-value problem for the corresponding system of ODEs with constant coefficients. The mapping (17) is calculated by solving the following initial-value problem, defined by the matrix $\boldsymbol{A}_{0}(163)$,

$$
\begin{array}{ll}
\frac{\mathrm{d} O}{\mathrm{~d} t}=-\alpha_{O U} O+\alpha_{P O} P, & O(0)=O_{n}, \\
\frac{\mathrm{d} P}{\mathrm{~d} t}=-\alpha_{P O} P+\alpha_{Q P} Q, & P(0)=P_{n}, \\
\frac{\mathrm{d} Q}{\mathrm{~d} t}=-\alpha_{Q P} Q+\alpha_{R Q} R, & Q(0)=Q_{n}, \\
\frac{\mathrm{d} R}{\mathrm{~d} t}=-\alpha_{R Q} R, & R(0)=R_{n}, \\
\frac{\mathrm{d} S}{\mathrm{~d} t}=-\alpha_{S T} S, & S(0)=S_{n}, \\
\frac{\mathrm{d} T}{\mathrm{~d} t}=\alpha_{S T} S-\alpha_{T U} T, & T(0)=T_{n}, \\
\frac{\mathrm{d} U}{\mathrm{~d} t}=\alpha_{O U} O+\alpha_{T U} T, & U(0)=U_{n}, \\
\frac{\mathrm{d} V}{\mathrm{~d} t}=0, & V(0)=V_{n}, \\
\frac{\mathrm{d} W}{\mathrm{~d} t}=0, & W(0)=W_{n},
\end{array}
$$

and then evaluating the result at $t=\Delta t$ to give $O_{n+1 / 3}, \ldots, W_{n+1 / 3}$. We note now that equations (169) and (170) are decoupled and we can solve them to get

$$
\begin{aligned}
& R(t)=R_{n} \mathrm{e}^{-\alpha_{R Q} t}, \\
& S(t)=S_{n} \mathrm{e}^{-\alpha_{S T} t} .
\end{aligned}
$$

We then substitute (175) into (168) to obtain a closed initial-value problem for $Q(t)$,

$$
\frac{\mathrm{d} Q}{\mathrm{~d} t}+\alpha_{Q P} Q=R_{n} \alpha_{R Q} \mathrm{e}^{-\alpha_{R Q} t}, \quad Q(0)=Q_{n},
$$

the solution of which is

$$
Q(t)=Q_{n} \mathrm{e}^{-\alpha_{Q P} t}-R_{n} \frac{\alpha_{R Q}\left(\mathrm{e}^{-\alpha_{Q P} t}-\mathrm{e}^{-\alpha_{R Q} t}\right)}{\alpha_{Q P}-\alpha_{R Q}} .
$$

Similarly, we substitute (176) into (171) to obtain

$$
T(t)=T_{n} \mathrm{e}^{-\alpha_{T U} t}-S_{n} \frac{\alpha_{S T}\left(\mathrm{e}^{-\alpha_{T U} t}-\mathrm{e}^{-\alpha_{S T} t}\right)}{\alpha_{T U}-\alpha_{S T}} .
$$

We then proceed in the same manner, by substituting the obtained solution (178) for $Q(t)$ into (167) to obtain $P(t)$, and the solution (180) for $T(t)$ into (172) to obtain $U(t)$, and finally the found solution for $P(t)$ into (166) to obtain $O(t)$. With the obvious solutions to (173) and (174), the result of all these steps is mapping

$$
\begin{aligned}
& O_{n+1 / 3}=\mu_{O U} O_{n}+K_{P O} P_{n}+K_{Q O} Q_{n}+K_{R O} R_{n}, \\
& P_{n+1 / 3}=\mu_{P O} P_{n}+K_{Q P} Q_{n}+K_{R P} R_{n}, \\
& Q_{n+1 / 3}=\mu_{Q P} Q_{n}+K_{R Q} R_{n}, \\
& R_{n+1 / 3}=\mu_{R Q} R_{n},
\end{aligned}
$$




$$
\begin{aligned}
& S_{n+1 / 3}=\mu_{S T} S_{n}, \\
& T_{n+1 / 3}=\mu_{T U} T_{n}+K_{S T} S_{n}, \\
& U_{n+1 / 3}=U_{n}+\left(1-\mu_{T U}\right) T_{n}+K_{S U} S_{n}+\left(1-\mu_{O U}\right) O_{n}+K_{P U} P_{n}+K_{Q U} Q_{n}+K_{R U} R_{n}, \\
& V_{n+1 / 3}=V_{n}, \\
& W_{n+1 / 3}=W_{n},
\end{aligned}
$$

where $\mu_{j k}=\mathrm{e}^{-\alpha_{j k} \Delta t}$ and

$$
\begin{aligned}
& K_{P O}=\frac{\alpha_{P O}\left(\mu_{P O}-\mu_{O U}\right)}{\alpha_{O U}-\alpha_{P O}}, \\
& K_{Q O}=\frac{\alpha_{P O} \alpha_{Q P}\left(\mu_{Q P}-\mu_{O U}\right)}{\left(\alpha_{P O}-\alpha_{Q P}\right)\left(\alpha_{O U}-\alpha_{Q P}\right)}-\frac{\alpha_{P O} \alpha_{Q P}\left(\mu_{P O}-\mu_{O U}\right)}{\left(\alpha_{P O}-\alpha_{Q P}\right)\left(\alpha_{O U}-\alpha_{P O}\right)}, \\
& K_{R O}=-\frac{\alpha_{P O} \alpha_{Q P} \alpha_{R Q}\left(\mu_{Q P}-\mu_{O U}\right)}{\left(\alpha_{Q P}-\alpha_{R Q}\right)\left(\alpha_{P O}-\alpha_{Q P}\right)\left(\alpha_{O U}-\alpha_{Q P}\right)}+\frac{\alpha_{P O} \alpha_{Q P} \alpha_{R Q}\left(\mu_{P O}-\mu_{O U}\right)}{\left(\alpha_{Q P}-\alpha_{R Q}\right)\left(\alpha_{P O}-\alpha_{Q P}\right)\left(\alpha_{O U}-\alpha_{P O}\right)}+, \\
& +\frac{\alpha_{P O} \alpha_{Q P} \alpha_{R Q}\left(\mu_{R Q}-\mu_{O U}\right)}{\left(\alpha_{Q P}-\alpha_{R Q}\right)\left(\alpha_{P O}-\alpha_{R Q}\right)\left(\alpha_{O U}-\alpha_{R Q}\right)}-\frac{\alpha_{P O} \alpha_{Q P} \alpha_{R Q}\left(\mu_{P O}-\mu_{O U}\right)}{\left(\alpha_{Q P}-\alpha_{R Q}\right)\left(\alpha_{P O}-\alpha_{R Q}\right)\left(\alpha_{O U}-\alpha_{P O}\right)}, \\
& K_{Q P}=\frac{\alpha_{Q P}\left(\mu_{Q P}-\mu_{P O}\right)}{\alpha_{P O}-\alpha_{Q P}} \\
& K_{R P}=-\frac{\alpha_{Q P} \alpha_{R Q}\left(\mu_{Q P}-\mu_{P O}\right)}{\left(\alpha_{Q P}-\alpha_{R Q}\right)\left(\alpha_{P O}-\alpha_{Q P}\right)}+\frac{\alpha_{Q P} \alpha_{R Q}\left(\mu_{R Q}-\mu_{P O}\right)}{\left(\alpha_{Q P}-\alpha_{R Q}\right)\left(\alpha_{P O}-\alpha_{R Q}\right)}, \\
& K_{R Q}=-\frac{\alpha_{R Q}\left(\mu_{Q P}-\mu_{R Q}\right)}{\alpha_{Q P}-\alpha_{R Q}}, \\
& K_{S T}=-\frac{\alpha_{S T}\left(\mu_{T U}-\mu_{S T}\right)}{\alpha_{T U}-\alpha_{S T}}, \\
& K_{S U}=1+\frac{\alpha_{S T} \mu_{T U}-\alpha_{T U} \mu_{S T}}{\alpha_{T U}-\alpha_{S T}} \\
& K_{P U}=1-\frac{\alpha_{O U} \mu_{P O}-\alpha_{P O} \mu_{O U}}{\alpha_{O U}-\alpha_{P O}}, \\
& K_{Q U}=\frac{\alpha_{P O}}{\alpha_{P O}-\alpha_{Q P}}\left(1-\frac{\alpha_{O U} \mu_{Q P}-\alpha_{Q P} \mu_{O U}}{\alpha_{O U}-\alpha_{Q P}}\right)-\frac{\alpha_{Q P}}{\alpha_{P O}-\alpha_{Q P}}\left(1-\frac{\alpha_{O U} \mu_{P O}-\alpha_{P O} \mu_{O U}}{\alpha_{O U}-\alpha_{P O}}\right), \\
& K_{R U}=-\frac{\alpha_{P O} \alpha_{R Q}}{\left(\alpha_{Q P}-\alpha_{R Q}\right)\left(\alpha_{P O}-\alpha_{Q P}\right)}\left(1-\frac{\alpha_{O U} \mu_{Q P}-\alpha_{Q P} \mu_{O U}}{\alpha_{O U}-\alpha_{Q P}}\right)+ \\
& +\frac{\alpha_{Q P} \alpha_{R Q}}{\left(\alpha_{Q P}-\alpha_{R Q}\right)\left(\alpha_{P O}-\alpha_{Q P}\right)}\left(1-\frac{\alpha_{O U} \mu_{P O}-\alpha_{P O} \mu_{O U}}{\alpha_{O U}-\alpha_{P O}}\right)+ \\
& +\frac{\alpha_{P O} \alpha_{Q P}}{\left(\alpha_{Q P}-\alpha_{R Q}\right)\left(\alpha_{P O}-\alpha_{R Q}\right)}\left(1-\frac{\alpha_{O U} \mu_{R Q}-\alpha_{R Q} \mu_{O U}}{\alpha_{O U}-\alpha_{R Q}}\right)- \\
& -\frac{\alpha_{Q P} \alpha_{R Q}}{\left(\alpha_{Q P}-\alpha_{R Q}\right)\left(\alpha_{P O}-\alpha_{R Q}\right)}\left(1-\frac{\alpha_{O U} \mu_{P O}-\alpha_{P O} \mu_{O U}}{\alpha_{O U}-\alpha_{P O}}\right) \text {. }
\end{aligned}
$$

At the second sub-step, the mapping (18) is calculated by solving the following initial-value problem, defined by the matrix $\boldsymbol{A}_{1}$ (164),

$$
\begin{aligned}
\frac{\mathrm{d} O}{\mathrm{~d} t}=-\alpha_{O P} O, & O(0) & =O_{n+1 / 3}, \\
\frac{\mathrm{d} P}{\mathrm{~d} t}=\alpha_{O P} O-\alpha_{P Q} P, & P(0) & =P_{n+1 / 3}, \\
\frac{\mathrm{d} Q}{\mathrm{~d} t}=\alpha_{P Q} P-\alpha_{Q R} Q, & Q(0) & =Q_{n+1 / 3}, \\
\frac{\mathrm{d} R}{\mathrm{~d} t}=\alpha_{Q R} Q, & R(0) & =R_{n+1 / 3}, \\
\frac{\mathrm{d} S}{\mathrm{~d} t}=\alpha_{T S} T, & S(0) & =S_{n+1 / 3}, \\
\frac{\mathrm{d} T}{\mathrm{~d} t}=\alpha_{U T} U-\alpha_{T S} T, & T(0) & =T_{n+1 / 3}, \\
\frac{\mathrm{d} U}{\mathrm{~d} t}=-\alpha_{U T} U, & & U(0)=U_{n+1 / 3},
\end{aligned}
$$




$$
\begin{array}{ll}
\frac{\mathrm{d} V}{\mathrm{~d} t}=0, & V(0)=V_{n+1 / 3}, \\
\frac{\mathrm{d} W}{\mathrm{~d} t}=0, & W(0)=W_{n+1 / 3}
\end{array}
$$

Here we proceed similar to the first sub-step. We note that the equations (202) and (208) are decoupled, and solve them to get $O(t)$ and $U(t)$. The result for $O(t)$ is substituted to (203) and the result for $U(t)$ is substituted to (207) to obtain closed initial value problems, which are solved to produce $P(t)$ and $T(t)$. The solution for $P(t)$ is substituted to the (204) to give $Q(t)$. Finally, we substitute the $Q(t)$ into (205) and $T(t)$ to (206) which yield $R(t)$ and $S(t)$ respectively. With the obvious solution to $V$ and $W$ the mapping is as follows:

$$
\begin{aligned}
& O_{n+2 / 3}=\mu_{O P} O_{n+1 / 3}, \\
& P_{n+2 / 3}=L_{O P} O_{n+1 / 3}+\mu_{P Q} P_{n+1 / 3}, \\
& Q_{n+2 / 3}=L_{O Q} O_{n+1 / 3}+L_{P Q} P_{n+1 / 3}+\mu_{Q R} Q_{n+1 / 3}, \\
& R_{n+2 / 3}=L_{O R} O_{n+1 / 3}+L_{P R} P_{n+1 / 3}+\left(1-\mu_{Q R}\right) Q_{n+1 / 3}+R_{n+1 / 3}, \\
& S_{n+2 / 3}=L_{U S} U_{n+1 / 3}+\left(1-\mu_{T S}\right) T_{n+1 / 3}+S_{n+1 / 3}, \\
& T_{n+2 / 3}=L_{U T} U_{n+1 / 3}+\mu_{T S} T_{n+1 / 3}, \\
& U_{n+2 / 3}=\mu_{U T} U_{n+1 / 3}, \\
& V_{n+2 / 3}=V_{n+1 / 3}, \\
& W_{n+2 / 3}=W_{n+1 / 3},
\end{aligned}
$$

where $\mu_{j k}=\mathrm{e}^{-\alpha_{j k} \Delta t}$ again, and

$$
\begin{aligned}
L_{O P} & =\frac{\alpha_{O P}\left(\mu_{O P}-\mu_{P Q}\right)}{\alpha_{P Q}-\alpha_{O P}}, \\
L_{O Q} & =\frac{\alpha_{P Q} \alpha_{O P}\left(\mu_{O P}-\mu_{Q R}\right)}{\left(\alpha_{P Q}-\alpha_{O P}\right)\left(\alpha_{Q R}-\alpha_{O P}\right)}-\frac{\alpha_{P Q} \alpha_{O P}\left(\mu_{P Q}-\mu_{Q R}\right)}{\left(\alpha_{P Q}-\alpha_{O P}\right)\left(\alpha_{Q R}-\alpha_{P Q}\right)}, \\
L_{P Q} & =\frac{\alpha_{P Q}\left(\mu_{P Q}-\mu_{Q R}\right)}{\alpha_{Q R}-\alpha_{P Q}}, \\
L_{O R} & =1+\frac{\alpha_{P Q}\left(\alpha_{O P} \mu_{Q R}-\alpha_{Q R} \mu_{O P}\right)}{\left(\alpha_{P Q}-\alpha_{O P}\right)\left(\alpha_{Q R}-\alpha_{O P}\right)}-\frac{\alpha_{O P}\left(\alpha_{P Q} \mu_{Q R}-\alpha_{Q R} \mu_{P Q}\right)}{\left(\alpha_{P Q}-\alpha_{O P}\right)\left(\alpha_{Q R}-\alpha_{P Q}\right)}, \\
L_{P R}=1+\frac{\alpha_{P Q} \mu_{Q R}-\alpha_{Q R} \mu_{P Q}}{\alpha_{Q R}-\alpha_{P Q}}, & \\
L_{U S}= & 1+\frac{\alpha_{U T} \mu_{T S}-\alpha_{T S} \mu_{U T}}{\alpha_{T S}-\alpha_{U T}}, \\
L_{U T}= & \frac{\alpha_{U T}\left(\mu_{U T}-\mu_{T S}\right)}{\alpha_{T S}-\alpha_{U T}} .
\end{aligned}
$$

The third sub-step mapping (19) is calculated by solving the following initial-value problem, defined by the matrix $\boldsymbol{A}_{2}$ (165),

$$
\begin{array}{llrl}
\frac{\mathrm{d} O}{\mathrm{~d} t}=\alpha_{U O} U, & O(0) & =O_{n+2 / 3}, \\
\frac{\mathrm{d} P}{\mathrm{~d} t}=\alpha_{U P} U-\alpha_{P U} P, & P(0) & =P_{n+2 / 3}, \\
\frac{\mathrm{d} Q}{\mathrm{~d} t}=\alpha_{T Q} T-\alpha_{Q T} Q, & Q(0) & =Q_{n+2 / 3}, \\
\frac{\mathrm{d} R}{\mathrm{~d} t}=\alpha_{S R} S-\alpha_{R S} R, & R(0) & =R_{n+2 / 3}, \\
\frac{\mathrm{d} S}{\mathrm{~d} t}=\alpha_{R S} R-\alpha_{S R} S, & S(0) & =S_{n+2 / 3}, \\
\frac{\mathrm{d} T}{\mathrm{~d} t}=\alpha_{Q T} Q-\alpha_{T Q} T, & T(0) & =T_{n+2 / 3}, \\
\frac{\mathrm{d} U}{\mathrm{~d} t}=\alpha_{P U} P+\alpha_{V U} V-\left(\alpha_{U P}+\alpha_{U O}+\alpha_{U V}\right) U, & U(0) & =U_{n+2 / 3}, \\
\frac{\mathrm{d} V}{\mathrm{~d} t}=\alpha_{U V} U+\alpha_{W V} W-\left(\alpha_{V U}+\alpha_{V W}\right) V, & V(0) & =V_{n+2 / 3}, \\
\frac{\mathrm{d} W}{\mathrm{~d} t}=\alpha_{V W} V-\alpha_{W V} W, & W(0) & =W_{n+2 / 3} .
\end{array}
$$


Unlike the previous sub-steps, this system is not solved exactly, but its solution is approximated by the forward Euler method as follows:

$$
\begin{aligned}
& O_{n+1}=O_{n+2 / 3}+\left(\alpha_{U O} U_{n+2 / 3}\right) \Delta t \\
& P_{n+1}=P_{n+2 / 3}+\left(\alpha_{U P} U_{n+2 / 3}-\alpha_{P U} P_{n+2 / 3}\right) \Delta t, \\
& Q_{n+1}=Q_{n+2 / 3}+\left(\alpha_{T Q} T_{n+2 / 3}-\alpha_{Q T} Q_{n+2 / 3}\right) \Delta t, \\
& R_{n+1}=R_{n+2 / 3}+\left(\alpha_{S R} S_{n+2 / 3}-\alpha_{R S} R_{n+2 / 3}\right) \Delta t, \\
& S_{n+1}=S_{n+2 / 3}+\left(\alpha_{R S} R_{n+2 / 3}-\alpha_{S R} S_{n+2 / 3}\right) \Delta t, \\
& T_{n+1}=T_{n+2 / 3}+\left(\alpha_{Q T} Q_{n+2 / 3}-\alpha_{T Q} T_{n+2 / 3}\right) \Delta t, \\
& U_{n+1}=U_{n+2 / 3}+\left[\alpha_{P U} P_{n+2 / 3}+\alpha_{V U} V_{n+2 / 3}-\left(\alpha_{U P}+\alpha_{U O}+\alpha_{U V}\right) U_{n+2 / 3}\right] \Delta t, \\
& V_{n+1}=V_{n+2 / 3}+\left[\alpha_{U V} U_{n+2 / 3}+\alpha_{W V} W_{n+2 / 3}-\left(\alpha_{V U}+\alpha_{V W}\right) V_{n+2 / 3}\right] \Delta t, \\
& W_{n+1}=W_{n+2 / 3}+\left(\alpha_{V W} V_{n+2 / 3}-\alpha_{W V} W_{n+2 / 3}\right) \Delta t .
\end{aligned}
$$

This completes the definition of the hybrid method.

\section{DETAILS OF ERROR ANALYSIS}

As proclaimed in the main text and as we shall see below, the local truncation errors at time step $\left[t_{n}, t_{n}+\Delta t\right]$ in all three methods are given by expressions of the form

$$
\mathcal{E}\left(t_{n}\right) \Delta t^{2}+\mathcal{O}\left(\Delta t^{3}\right)
$$

giving the upper estimate of a global error for the interval $t \in\left[t_{\min }, t_{\max }\right]$ of the first order,

$$
\sup _{\left[t_{\min }, t_{\max }\right]}\left\|\vec{u}^{\text {exact }}-\vec{u}^{\text {numeric }}\right\| \leq \sup _{\left[t_{\min }, t_{\max }\right]}(\mathcal{E}(t))\left(t_{\max }-t_{\min }\right) \Delta t+\mathcal{O}\left(\Delta t^{2}\right),
$$

where the estimates of the coefficients $\mathcal{E}$ are different for the three methods.

To obtain these estimates, let us consider the quasi-linear system (9), rewritten as

$$
\frac{\mathrm{d} \vec{u}}{\mathrm{~d} t}=\boldsymbol{A}\left(V_{m}(t)\right) \vec{u}=\boldsymbol{A}(t) \vec{u}
$$

on the interval $t \in\left[t_{n}, t_{n+1}\right], t_{n+1}=t_{n}+\Delta t$. Using matrix exponential, the result can be written in the form

$$
\vec{u}\left(t_{n+1}\right)=\exp \left[\int_{t_{n}}^{t_{n}+\Delta t} \boldsymbol{A}\left(t^{\prime}\right) \mathrm{d} t^{\prime}\right] \vec{u}\left(t_{n}\right) \equiv \boldsymbol{T}\left(t_{n}, \Delta t\right) \vec{u}\left(t_{n}\right)
$$

The accuracy in finding $\vec{u}\left(t_{n+1}\right)$ at a given $\vec{u}\left(t_{n}\right)$ depends on accuracy of the approximation of operator $\boldsymbol{T}$ and on the norm of vector $\vec{u}\left(t_{n}\right)$. Since each component of $\vec{u}$ is restricted to the interval $[0,1]$ and sum of its components is fixed to 1 , we have $\|\vec{u}\| \leq 1$ for any choice of norm, in which any vector, that has exactly one component equal to unity and the rest equal to zero, is a unit vector.

Hence from this point on we focus on the approximation of the timestep transition operator $\boldsymbol{T}$.

Expanding (247), first the integral, then the exponential, in the Taylor series, we have

$$
\begin{aligned}
\boldsymbol{T} & =\exp \left[\int_{t_{n}}^{t_{n}+\Delta t}\left(\boldsymbol{A}\left(t_{n}\right)+\dot{\boldsymbol{A}}\left(t_{n}\right)\left(t^{\prime}-t_{n}\right)+\mathcal{O}\left(\left(t^{\prime}-t_{n}\right)^{2}\right)\right) \mathrm{d} t^{\prime}\right]=\exp \left[\boldsymbol{A}\left(t_{n}\right) \Delta t+\frac{1}{2} \dot{\boldsymbol{A}}\left(t_{n}\right) \Delta t^{2}+\mathcal{O}\left(\Delta t^{3}\right)\right] \\
& =1+\left[\boldsymbol{A}\left(t_{n}\right) \Delta t+\frac{1}{2} \dot{\boldsymbol{A}}\left(t_{n}\right) \Delta t^{2}+\mathcal{O}\left(\Delta t^{3}\right)\right]+\frac{1}{2}\left[\boldsymbol{A}\left(t_{n}\right) \Delta t+\frac{1}{2} \dot{\boldsymbol{A}}\left(t_{n}\right) \Delta t^{2}+\mathcal{O}\left(\Delta t^{3}\right)\right]^{2}+\mathcal{O}\left(\Delta t^{3}\right) \\
& =1+\boldsymbol{A}\left(t_{n}\right) \Delta t+\frac{1}{2}\left[\dot{\boldsymbol{A}}\left(t_{n}\right)+\boldsymbol{A}^{2}\left(t_{n}\right)\right] \Delta t^{2}+\mathcal{O}\left(\Delta t^{3}\right),
\end{aligned}
$$

where the dot designates time differentiation. FE approximates this operator as

$$
\boldsymbol{T}_{\mathrm{EF}}\left(t_{n}, \Delta t\right)=1+\boldsymbol{A}\left(t_{n}\right) \Delta t
$$

hence for the principal term of the norm of the error we have

$$
\mathcal{E}_{\mathrm{FE}}=\lim _{\Delta t \rightarrow 0}\left\|\boldsymbol{T}_{\mathrm{EF}}-\boldsymbol{T}\right\| / \Delta t^{2}=\frac{1}{2}\left\|\boldsymbol{A}^{2}+\dot{\boldsymbol{A}}\right\| \leq \frac{1}{2}\left(\|\boldsymbol{A}\|^{2}+\|\dot{\boldsymbol{A}}\|\right) .
$$

For the MRL, we have

$$
\boldsymbol{T}_{\mathrm{MRL}}=\exp \left(\boldsymbol{A}\left(t_{n}\right) \Delta t\right)=1+\boldsymbol{A}\left(t_{n}\right) \Delta t+\frac{1}{2} \boldsymbol{A}^{2}\left(t_{n}\right) \Delta t^{2}+\mathcal{O}\left(\Delta t^{3}\right),
$$




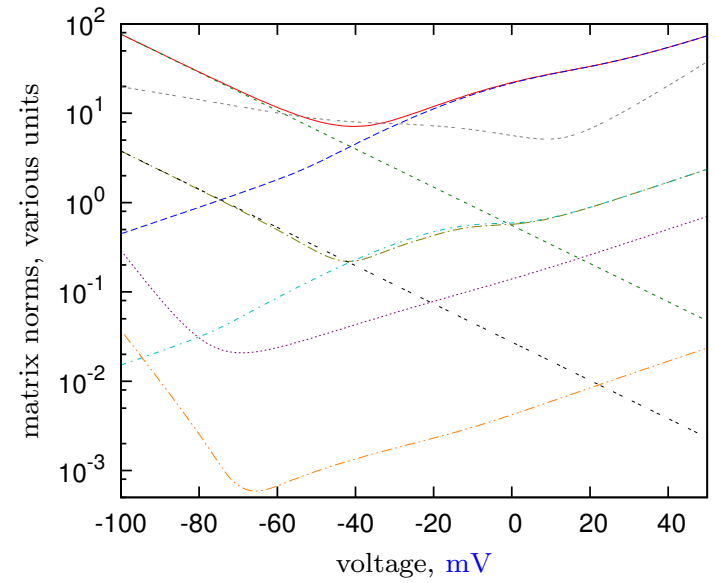

(a)

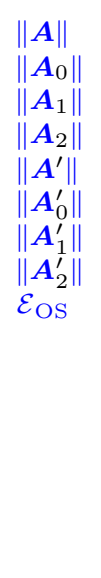

(b)

Fig. 6. (a) Matrix norms affecting the apriori error estimates, as functions of the transmembrane voltage. Norms $\|\boldsymbol{A}\|,\left\|\boldsymbol{A}_{m}\right\|$ are in ms ${ }^{-1},\left\|\boldsymbol{A}^{\prime}\right\|,\left\|\boldsymbol{A}_{m}^{\prime}\right\|, \mathcal{E}_{\mathrm{OS}}$ are in $\mathrm{ms}^{-2}$. (b) The coefficients of the apriori error estimates, as functions of time. $\mathcal{E}_{\mathrm{OS}}$ component shown by points as it overlaps with $\mathcal{E}_{\mathrm{HOS}}$ most of the time. The action potential $V_{m}(t)$ is shown for reference.

therefore

$$
\mathcal{E}_{\mathrm{MRL}}=\frac{1}{2}\|\dot{\boldsymbol{A}}\|=\frac{1}{2}\left\|\boldsymbol{A}^{\prime}\right\|\left|\dot{V}_{m}\right|
$$

where the prime designates differentiation by $V_{m}$.

The errors of the three substeps of HOS are described by the above formulas for FE (for $\boldsymbol{A}_{2}$ ) and for MRL (for $\boldsymbol{A}_{0}, \boldsymbol{A}_{1}$ ), and in addition to those, we have the error due to operator splitting. To estimate the latter, let us compare the exact solution with the result of the successive application of the substeps as if they were done exactly. Let $\boldsymbol{B}_{m}=\int_{t_{n}}^{t_{n}+\Delta t} \boldsymbol{A}_{m}(t) \mathrm{d} t, m=0,1,2$. Then the exact solution is

$$
\begin{aligned}
\boldsymbol{T} & =1+\left(\boldsymbol{B}_{0}+\boldsymbol{B}_{1}+\boldsymbol{B}_{2}\right)+\frac{1}{2}\left(\boldsymbol{B}_{0}+\boldsymbol{B}_{1}+\boldsymbol{B}_{2}\right)^{2}+\mathcal{O}\left(\Delta t^{3}\right) \\
& =1+\left(\boldsymbol{B}_{0}+\boldsymbol{B}_{1}+\boldsymbol{B}_{2}\right)+\frac{1}{2}\left(\boldsymbol{B}_{0}^{2}+\boldsymbol{B}_{1}^{2}+\boldsymbol{B}_{2}^{2}+\boldsymbol{B}_{0} \boldsymbol{B}_{1}+\boldsymbol{B}_{1} \boldsymbol{B}_{0}+\boldsymbol{B}_{0} \boldsymbol{B}_{2}+\boldsymbol{B}_{2} \boldsymbol{B}_{0}+\boldsymbol{B}_{1} \boldsymbol{B}_{2}+\boldsymbol{B}_{2} \boldsymbol{B}_{1}\right)+\mathcal{O}\left(\Delta t^{3}\right)
\end{aligned}
$$

and the result of the three substeps, with $\mathrm{e}^{\boldsymbol{B}_{0}}$ applied first and $\mathrm{e}^{\boldsymbol{B}_{2}}$ applied last, is

$$
\begin{aligned}
\boldsymbol{T}_{\mathrm{OS}} & =\left(1+\boldsymbol{B}_{2}+\frac{1}{2} \boldsymbol{B}_{2}^{2}+\mathcal{O}\left(\Delta t^{3}\right)\right)\left(1+\boldsymbol{B}_{1}+\frac{1}{2} \boldsymbol{B}_{1}^{2}+\mathcal{O}\left(\Delta t^{3}\right)\right)\left(1+\boldsymbol{B}_{0}+\frac{1}{2} \boldsymbol{B}_{0}^{2}+\mathcal{O}\left(\Delta t^{3}\right)\right) \\
& =1+\boldsymbol{B}_{2}+\boldsymbol{B}_{1}+\boldsymbol{B}_{0}+\frac{1}{2}\left(\boldsymbol{B}_{2}^{2}+\boldsymbol{B}_{1}^{2}+\boldsymbol{B}_{0}^{2}+2 \boldsymbol{B}_{2} \boldsymbol{B}_{1}+2 \boldsymbol{B}_{2} \boldsymbol{B}_{0}+2 \boldsymbol{B}_{1} \boldsymbol{B}_{0}\right)+\mathcal{O}\left(\Delta t^{3}\right)
\end{aligned}
$$

so

$$
\boldsymbol{T}_{\mathrm{OS}}-\boldsymbol{T}=\frac{1}{2}\left(\left[\boldsymbol{B}_{2}, \boldsymbol{B} 1\right]+\left[\boldsymbol{B}_{2}, \boldsymbol{B}_{0}\right]+\left[\boldsymbol{B}_{1}, \boldsymbol{B}_{0}\right]\right)+\mathcal{O}\left(\Delta t^{3}\right)=\frac{1}{2}\left(\left[\boldsymbol{A}_{2}, \boldsymbol{A} 1\right]+\left[\boldsymbol{A}_{2}, \boldsymbol{A}_{0}\right]+\left[\boldsymbol{A}_{1}, \boldsymbol{A}_{0}\right]\right) \Delta t^{2}+\mathcal{O}\left(\Delta t^{3}\right)
$$

where we use the standard notation for the matrix commutator, $[\boldsymbol{X}, \boldsymbol{Y}] \equiv \boldsymbol{X} \boldsymbol{Y}-\boldsymbol{Y} \boldsymbol{X}$. Finally, by the triangle inequality (subadditivity) of a matrix norm, the upper estimate of the error coefficient $\mathcal{E}_{\mathrm{HOS}}$ is given by the sum of the error coefficients of the three constituent steps and of the operator splitting.

To summarize, we have the following estimates of the leading terms of the approximation errors for the three methods as

$$
\begin{aligned}
& \mathcal{E}_{\mathrm{FE}} \leq \frac{1}{2}\left(\|\boldsymbol{A}\|^{2}+\left\|\mathrm{d} \boldsymbol{A} / \mathrm{d} V_{m}\right\|\left|\mathrm{d} V_{m} / \mathrm{d} t\right|\right) \\
& \mathcal{E}_{\mathrm{MRL}}=\frac{1}{2}\left\|\mathrm{~d} \boldsymbol{A} / \mathrm{d} V_{m}\right\|\left|\mathrm{d} V_{m} / \mathrm{d} t\right| \\
& \mathcal{E}_{\mathrm{HOS}} \leq \frac{1}{2}\left|\mathrm{~d} V_{m} / \mathrm{d} t\right|\left(\left\|\mathrm{d} \boldsymbol{A}_{0} / \mathrm{d} V_{m}\right\|+\left\|\mathrm{d} \boldsymbol{A}_{1} / \mathrm{d} V_{m}\right\|+\left\|\mathrm{d} \boldsymbol{A}_{2} / \mathrm{d} V_{m}\right\|\right)+\frac{1}{2}\left\|\boldsymbol{A}_{2}\right\|^{2}+\mathcal{E}_{\mathrm{OS}}, \\
& \mathcal{E}_{\mathrm{OS}}=\frac{1}{2}\left\|\left[\boldsymbol{A}_{1}, \boldsymbol{A}_{0}\right]+\left[\boldsymbol{A}_{2}, \boldsymbol{A}_{0}\right]+\left[\boldsymbol{A}_{2}, \boldsymbol{A}_{1}\right]\right\| .
\end{aligned}
$$


An important observation is that the apriori estimates of the errors cannot be made based on the properties of the MC alone as they depend on the rate of change of the voltage.

The graphs of the Frobenius norms of the matrices involved in the estimates (248) are shown in Fig. 6(a). Evidently $\|\boldsymbol{A}\|$ dominates other norms throughout the voltage range; however, it is relatively small for intermediate values of $V_{m}$ and this is precisely when $\mathrm{d} V_{m} / \mathrm{d} t$ is large during a typical AP, making the related components of the errors more significant. So a more adequate idea of the relative magnitudes of the errors of the three methods should take into account properties of specific solutions. Figure 6(b) shows the values of the error estimates (248) for the typical AP which was used for other numerical illustrations in the paper. We see that the error associated with FE is the largest of the three, with the maximal magnitude of about $2700 \mathrm{~ms}^{-2}$, achieved early during the plateau of the AP, thus guaranteeing no more than $10 \%$ global error on a time interval of $1 \mathrm{~ms}$ long for time steps as short as $\Delta t \approx 0.04 \mu \mathrm{s}$, and its main contributor is $\|\boldsymbol{A}\|^{2}$ rather than $\|\dot{\boldsymbol{A}}\|$. The error associated with MRL is the smallest of the three, with the maximal magnitude of about $118 \mathrm{~ms}^{-2}$, achieved during the upstroke of the action potential, giving $10 \%$ global accuracy on $1 \mathrm{~ms}$ interval for $\Delta t \approx 0.8 \mu \mathrm{s}$. The error of the HOS is intermediate between the two. Its maximum of about $125 \mathrm{~ms}^{-2}$, i.e. very similar to that of MRL and achieved at the same time, as its main contributors are the same $\dot{V}_{m}$-dependent errors of the exponential integrator substeps as in $\mathcal{E}_{\mathrm{MRL}}$. Outside the AP upstroke, the error of HOS is dominated by the operator splitting error $\mathcal{E}_{\mathrm{OS}}$, which however never exceeds $19 \mathrm{~ms}^{-2}$. The ratio of the error coefficients of the two methods varies widely during the AP solution: $\mathcal{E}_{\mathrm{FE}} / \mathcal{E}_{\mathrm{MRL}} \in(3.18, \infty)$ (remember $\mathcal{E}_{\mathrm{MRL}}=0$ when $\left.\mathrm{d} V_{m} / \mathrm{d} t=0\right)$ and $\mathcal{E}_{\mathrm{FE}} / \mathcal{E}_{\mathrm{HOS}} \in(2.30,161)$, with the smallest values achieved during the upstroke when the exponential solvers are least accurate.

Clearly, the estimate of the global error given by (246) is over-cautious, or "pessimistic", as it presumes that local errors take maximal values allowed by the matrix norms, and accumulate but not compensate on the whole interval $\left[t_{\min }, t_{\max }\right]$. As the numerical experiments described in the main text show, the actual errors are much smaller. Still, the analysis done here can be useful in identifying relative contribution of different sources of errors and identifying "bottlenecks". Specifically, we see that

- the exponential solvers are more accurate than FE: even in the worst case, during the upstroke, they give two to three times smaller error;

- the principal limitation of the accuracy of both exponential solvers is the dependence on $\dot{V}_{m}$, which affects accuracy mostly during the upstroke, hence any attempts to improve the accuracy should in the first instance address this issue.

\section{REFERENCES}

[1] A. L. Hodgkin and A. F. Huxley, "A quantitative description of membrane current and its application to conduction and excitation in nerve," J. Physiol, vol. 117, no. 4, pp. 500-544, 1952.

[2] R. L. Burden and J. D. Faires, Numerical Analysis, 9th ed. Boston: Brooks/Cole, 2011.

[3] S. Rush and H. Larsen, "A practical algorithm for solving dynamic membrane equations," IEEE Trans. Biomed. Eng., vol. 25, no. 4, pp. 389-392, 1978.

[4] J. Sundnes, R. Artebrant, O. Skavhaug, and A. Tveito, "A second-order algorithm for solving dynamic cell membrane equations," IEEE Trans. Biomed. Eng., vol. 56, no. 10, pp. 2546-2548, 2009.

[5] M. Perego and A. Veneziani, "An efficient generalization of the Rush-Larsen method for solving electro-physiology membrane equations," Electronic Transactions on Numerical Analysis, vol. 35, pp. 234-256, 2009.

[6] M. E. Marsh, S. T. Ziaratgahi, and R. J. Spiteri, "The secrets to the success of the Rush-Larsen method and its generalizations," IEEE Trans. Biomed. Eng., vol. 59, no. 9, pp. 2506-2515, 2012.

[7] A. Reidman and K. Trivedi, "Numerical transient analysis of Markov models," Comput. Opns Res., vol. 15, no. 1, pp. 19-36, 1988.

[8] C. E. Clancy and Y. Rudy, "Na ${ }^{+}$channel mutation that causes both Brugada and long-QT syndrome phenotypes: a simulation study of mechanism," Circulation, vol. 105, no. 10, pp. 1208-1213, 2002.

[9] C. H. Luo and Y. Rudy, "A dynamic-model of the cardiac ventricular action-potential. 1. Simulations of ionic currents and concentration changes," Circulation Research, vol. 74, no. 6, pp. 1071-1096, 1994.

[10] J. L. Zeng, K. R. Laurita, D. S. Rosenbaum, and Y. Rudy, "Two components of the delayed rectifier $\mathrm{K}^{+}$current in ventricular myocytes of the guinea-pig type. theoretical formulation and their role in repolarization," Circulation Research, vol. 77, no. 1, pp. 140-152, 1995.

[11] P. C. Viswanathan, R. M. Shaw, and Y. Rudy, "Effects of $I_{K r}$ and $I_{K s}$ heterogeneity on action potential duration and its rate dependence. a simulation study," Circulation, vol. 99, no. 18, May 1999.

[12] W. Stein et al., Sage Mathematics Software (Version 5.9), The Sage Development Team, 2013, http://www.sagemath.org. 